\title{
ESSENTIAL CENTRAL RANGE AND SELFADJOINT COMMUTATORS IN PROPERLY INFINITE VON NEUMANN ALGEBRAS $\left({ }^{\mathbf{1}}\right)$
}

BY

\author{
HERBERT HALPERN
}

\begin{abstract}
The essential central range of an element $A$ of a von Neumann algebra with respect to a central ideal is characterized as those elements arbitrarily close to the compression of $A$ to a subspace large with respect to the ideal. The selfadjoint commutators in a properly infinite algebra are shown to be the elements whose essential central ranges with respect to the strong radical contain 0 .
\end{abstract}

1. Introduction. Let $\mathscr{Q}$ be a von Neumann algebra with center $\mathscr{\mathscr { L }}$ Let ( () denote the set of nonzero projections in a subset $\mathcal{S}$ of $\mathbb{Q}$. A (closed two-sided) ideal $\mathscr{G}$ of $\mathscr{Q}$ is said to be a central ideal of $\mathscr{Q}$ if given any bounded set $\left\{A_{i}\right\}$ in $\mathscr{G}$ and a corresponding set $\left\{P_{i}\right\}$ of orthogonal projections in (Z) then the operator $\sum A_{i} P_{i}$ is in 9 . For an element $\zeta$ of the spectrum $Z$ of $\mathscr{L}$, let [ $\zeta$ ] denote the smallest ideal of $\mathscr{Q}$ containing $\zeta$. Then the ideal $g$ is a central ideal if and only if the map

$$
\zeta \rightarrow\|A(g+[\zeta])\|=\operatorname{glb}\{\|A+B\| \mid B \in \mathcal{G}+[\zeta]\}
$$

is continuous on $Z$ for every $A$ in $Q$. If $P$ is a projection in $\mathscr{Z}$ and if $E$ is a properly infinite projection in $Q P$ (by convention 0 is properly infinite), then the set of projections $F$ in $Q P$ such that $Q F>E Q$ for some central projection $Q$ implies $E Q=0$ is the set of all projections of a central ideal denoted by $g_{P}(E)$. All central ideals are of this form. If $Q P$ is the weak closure of the central ideal, then the form is canonical in the sense that $g_{P}(E)=g_{Q}(F)$ if and only if $P=Q$ and $E \sim F$ [12].

Now let $\mathscr{Q}$ be a central ideal of $Q$. If $A$ is an element in $Q$, there is a largest central projection $c_{g}(A)$ in $\mathscr{Q}$ such that $c_{g}(A) A$ is in 9 . The projection $1-c_{g}(1)$ is denoted by $P_{g}$. A projection $F$ in $\mathbb{Q}$ is said to have dimension greater than the central ideal $\mathscr{G}=g_{P}(E)$ (in symbols, $\operatorname{dim} F>\operatorname{dim} G$ ) if $F$ has central support $P_{g}$ and if $F \succ E P_{g}$ or equivalently, if $F$ has central support $P_{g}$ and $c_{g}(F)$

Received by the editors January 22, 1975.

AMS (MOS) subject classifications (1970). Primary 46L10, $47 \mathrm{C} 15$.

Key words and phrases. Essential central spectrum, selfadjoint commutators.

(1)This research was supported by the National Science Foundation.

() American Mathematic:al Suciety 1977 
$=1-P_{g}$. The essential central spectrum $\mathscr{Z}-\mathrm{Sp}_{g} A$ of an element $A$ in $\mathbb{Q}$ modulo the central ideal $g$ is the set of all $C$ in $\mathscr{Z}$ such that $C^{\wedge}(\zeta)$ is in the spectrum of the image $A(g+[\zeta])$ of $A$ in $\mathscr{Q} /(g+[\zeta])$ under the canonical homomorphism for every $\zeta$ in $Z$. Here $C^{\wedge}$ is the Gelfand transform of $C$. If $g$ is the ideal (0), then the essential central spectrum of $A$ modulo $g$ is called the central spectrum of $A$ and is written $\mathscr{Z}-\mathrm{Sp} A$. The set $\mathscr{Z}-\mathrm{Sp}_{9} A$ is nonvoid and strongly closed. Let $\mathbb{Q}^{\sim}$ be the Banach space of all bounded $\mathscr{L}$-module homomorphisms of $\mathscr{Q}$ into $\mathcal{Z}$ and let $\mathbb{Q}^{\sim+}$ be the subset of all those $\phi$ in $\mathbb{Q}^{\sim}$ that $\operatorname{map} \mathbb{Q}^{+}$into $\mathbb{Z}^{+}$. An element $\phi$ in $\mathbb{Q}^{\sim+}$ is called a state in $\mathbb{Q}^{\sim}$ if $\phi(1)=1$. For example, if $E$ is a maximal abelian projection (i.e., $E$ has central support 1 ) in the commutant $\mathscr{Z}^{\prime}$ of $\mathscr{Z}$ and if $\tau_{E}(A)$ denotes the unique element in $\mathscr{Z}$ with $\tau_{E}(A) E=E A E$ for $A$ in $\mathscr{Q}$, then the map $\tau_{E}$ is a state of $\mathbb{Q}^{\sim}$. Let $E_{a}(q)$ be the subset of $\mathcal{Q}^{\sim+}$ given by

$$
E_{a}(\mathcal{G})=\left\{\phi \in \mathbb{Q}^{\sim+} \mid \phi(\mathcal{G})=0, \phi\left(P_{\mathcal{g}}\right)=P_{\mathcal{G}}\right\} .
$$

The essential central range $\Re_{g}(A)$ of an element $A$ in $\mathscr{Q}$ modulo the central ideal $g$ is defined to be the set

$$
\mathfrak{K}_{g}(A)=\left\{\phi(A) \mid \phi \in E_{a}(\mathcal{g})\right\} .
$$

The set $\mathscr{K}_{g}(A)$ is also equal to the set

$$
\cap\left\{\text { unif clos }\left\{\tau_{E}(A+B) \mid E \text { abelian of central support } P_{g}\right\} \mid B \in g\right\}
$$

$[12,4.8]$, and is a weakly closed $\mathscr{Z}$-convex subset of $\mathscr{Z}$ containing $\mathscr{Z}-\operatorname{Sp}_{\mathrm{g}} A$. Here a set $\delta$ is $\mathscr{L}$-convex if $C A_{1}+(1-C) A_{2}$ is in $\delta$ whenever $C$ is in $\mathscr{Z}$ with $0 \leqslant C \leqslant 1$ and the $A_{i}$ are in $\delta$ [12].

If $Q$ is a finite von Neumann algebra, then every central ideal is of the form $\mathbb{Q}$ for some central projection $P$. If $\mathbb{Q}$ is a properly infinite von Neumann algebra, then the ideal $g_{0}$ generated by the finite projections is a central ideal and $g_{0}$ has canonical form

$$
g_{0}=g_{1}(E)
$$

where $E$ is a projection of central support 1 for which there is an orthogonal set $\left\{P_{i}\right\}$ in (Q) of sum 1 such that $E P_{i}$ is a $\sigma$-finite projection. If $Q$ is the set of all bounded operators on a Hilbert space, then $g_{0}$ is the ideal of compact operators. In general, we have that $\mathscr{K}_{g_{0}}(A)$ is equal to the intersection of $\mathscr{Z}$ with the weak closure of the convex hull of the set $U A U^{*}$ where $U$ runs through the set $U(\mathbb{Q})$ of unitary operators in $\mathbb{Q}$. The strong radical $g$ of $\mathscr{Q}$ (i.e., the intersection of all maximal ideals of $\mathbb{Q}$ ) is also a central ideal of $\mathscr{Q}$ and $g$ may be written as $g=g_{1}(1)$. In particular, the dimension of the projection $E$ is greater than that of $g$ if and only if $E \sim 1$. In this case the set $\Re_{q}(A)$ is the 
intersection of $\mathscr{Z}$ with the uniform closure of the convex hull of $\left\{U A U^{*} \mid U\right.$ $\in U(\mathbb{Q})\}$. In the sequel, we write $\Re_{g}(A)$ simply as $\mathscr{K}(\mathbb{Q})$ [10], [12].

In this note we show that $C$ is in the essential central range of $A$ modulo the central ideal $g$ if and only if there is, for every $\varepsilon>0$, a projection $E$ in $Q$ with $\operatorname{dim} E>\operatorname{dim} 9$ such that $\|E(C-A) E\|<\varepsilon$. For a properly infinite algebra $\mathbb{Q}$, the operator $C$ is in $\mathscr{K}_{g}(A)$ if and only if there is a projection $E$ in $\mathbb{Q}$ with $\operatorname{dim} E>\operatorname{dim} 9$ such that $C E$ is in the essential central spectrum of $E A E$ in the algebra $\mathfrak{Q}_{E}$ modulo the central ideal $g \cap \mathfrak{Q}_{E}$. Here $\mathfrak{Q}_{E}$ is the von Neumann algebra $E Q E$ on the Hilbert space determined by $E$. Also the set $\mathscr{K}_{g_{0}}(A)$ is shown to be the intersection of the center with the weak closure of $\left\{U A U^{*} \mid U \in U(\mathbb{Q})\right\}$. Using this characterization of the essential central range, we show by displaying a matrix form similar to that given by J. Anderson [1] that an element $A$ in a properly infinite von Neumann algebra $Q$ is a selfadjoint commutator in $\mathbb{Q}$ (i.e., there are $B$ and $C$ in $\mathscr{Q}$ with $A=B C-C B$ and $B=B^{*}$ ) if and only if 0 is in $\Re(A)$. We also characterize operators similar to selfadjoint commutators. Finally, we discuss the problem of characterizing commutators in properly infinite von Neumann algebras.

2. Characterization of the essential central range. In this section we show that a central element $C$ is in the essential range of $A$ modulo a central ideal $g$ if and only if the compression of $C-A$ to a subspace that is large with respect to $g$ is small.

LEMma 2.1. Let $C$ be in the essential central range of an element $A$ in a continuous von Neumann algebra $\mathbb{Q}$ modulo the central ideal $\mathcal{G}$ of $\mathbb{Q}$; then there is, for every $\varepsilon>0$, an extreme point $\phi$ of $E_{a}(9)$ such that $\|\phi(A)-C\|<\varepsilon$ and such that the kernel of the canonical representation of the positive functional $\phi_{\zeta}$ given by $\phi_{\zeta}(B)=\phi(B)^{\wedge}(\zeta)$ is $G+[\zeta]$ for every $\zeta$ in the spectrum $Z$ of the center $\mathcal{Z}$ of $Q$.

PRoof. By performing a preliminary reduction, we may assume that $P_{g}=1$ so that the canonical representation $g_{P}(E)$ of $g$ has the property that the central support of $E$ is $P[11,2.7]$. The set $\delta$ of all positive $\mathcal{L}$-module homomorphisms $\psi$ of $\mathbb{Q}$ into $\mathscr{Z}$ such that $\psi(\mathscr{g})=(0)$ and $\psi(E)=P$ is a nonvoid, $\mathscr{L}$-convex subset of $\mathbb{Q}^{\sim+}$ that is compact in the topology of pointwise convergence on $\mathscr{Q}$ where $\mathcal{Z}$ is taken with the weak operator topology $[12,4.3]$. If $\psi_{1}$ is an extreme point of $\delta$ and if $\psi_{2}$ is a pure (i.e., extreme) state of $\mathbb{Q}^{\sim+}$ whose canonical representation is faithful $[9,4.6]$, then the $\mathcal{E}$-module homomorphism $\phi=P \psi_{1}+(1-P) \psi_{2}$ is a pure state of $\mathbb{Q}^{\sim+}$. We show that the kernel of the canonical representation $\pi_{\zeta}$ induced by the state $\varphi_{\zeta}$ is $g+[\zeta]$. On the one hand, if $P^{\wedge}(\zeta)=0$, then $\pi_{\zeta}(B)=0$ if and only if

$$
\left(\psi_{2}\right)_{\zeta}\left(D^{*} B D\right)=\phi_{\zeta}\left(D^{*} B D\right)=0
$$


for all $D$ in $Q$, or equivalently, if and only if $B$ is in the kernel $[\zeta]=g+[\zeta]$ of the canonical representation of $\left(\psi_{2}\right)_{\zeta}$ [9, Theorem 4.7]. On the other hand, if $P^{\wedge}(\zeta)=1$, then it is clear that the ideal $g+[\zeta]$ is contained in the kernel of $\pi_{\xi}$. Now supposing $B$ is not in $g+[\zeta]$, we may find a spectral projection $F$ of $B^{*} B$ corresponding to an interval of the form $\left[\alpha,\|B\|^{2}\right]$ with $\alpha>0$ such that $F$ is not in $g+[\zeta]$. We have that $c_{g}(F)^{\wedge}(\zeta)=0$; otherwise, the projection

$$
F=c_{g}(F) F+\left(1-c_{g}(F)\right) F
$$

is in $g+[\zeta]$. Because

$$
\left(1-c_{g}(F)\right) F \succ\left(1-c_{g}(F)\right) E,
$$

there is a partial isometry $U$ in $\mathscr{Q}$ with $U^{*} U=\left(1-c_{g}(F)\right) E$ and $U U^{*}$ $\leqslant\left(1-c_{g}(F)\right) F$. The fact that

$$
\phi_{\zeta}\left(U^{*} U\right)=\phi_{\zeta}(E)=1
$$

implies that $\pi_{\zeta}(U) \neq 0$ and consequently that

$$
\pi_{\zeta}\left(B^{*} B\right) \geqslant \alpha \pi_{\zeta}(F) \geqslant \alpha \pi_{\zeta}\left(U U^{*}\right)>0 .
$$

This proves that the kernel of $\pi_{\zeta}$ is $g+[\zeta]$.

Now let $C$ be in $\mathscr{K}_{g}(A)$. There is a $\psi$ in $E_{a}(\mathcal{g})$ with $\psi(A)=C$. For every $\zeta$ in $Z$, there is a state $\theta_{\zeta}$ of $\pi_{\zeta}(Q)$ such that $\psi_{\zeta}=\theta_{\zeta} \pi_{\zeta}$. Since $\pi_{\zeta}$ is an irreducible representation of the continuous algebra $\mathbb{Q}$ [9, Corollary, Theorem 4.3], there is a unitary operator $U$ in $\mathbb{Q}$ such that

$$
\left|\theta_{\zeta}\left(\pi_{\xi}(A)\right)-\left(\pi_{\zeta}(A) \pi_{\zeta}(U) x_{\zeta}, \pi_{\zeta}(U) x_{\zeta}\right)\right|<\varepsilon
$$

[8, Theorem 2]. Here $x_{\zeta}$ denotes a unit vector in the Hilbert space of $\pi_{\zeta}$ such that $\left(\pi_{\xi}(B) x_{\zeta}, x_{\xi}\right)=\phi_{\xi}(B)$ for every $B$ in $Q$. Due to the continuity of the left hand side of (1) as a function of $\zeta$, we may use the fact that $Z$ is extremally disconnected to find a unitary $U$ in $\mathbb{Q}$ such that $\left\|\psi(A)-\phi\left(U^{*} A U\right)\right\|<\varepsilon$. The state $B \rightarrow \phi\left(U^{*} B U\right)^{\wedge}(\zeta)$ of $Q$ is a pure state whose canonical representation has kernel $g+[\zeta]$. Q.E.D.

We now give a characterization of the essential central range. This was previously known only for selfadjoint elements [12, 4.9].

THEOREM 2.2. In order that an element $C$ in the center $\mathcal{Z}$ of a von Neumann algebra $\mathbb{Q}$ be in the essential central range of an element $A$ in $\mathbb{Q}$ with respect to the central ideal 9 , a necessary and sufficient condition is that $C P_{9}=C$ and that, for every $\varepsilon>0$, there is a projection $F$ in $\mathbb{Q}$ with $\operatorname{dim} F>\operatorname{dim} G$ such that $\|F(C-A) F\|<\varepsilon$. 
Proof. Suppose first that $C$ is in $\nVdash_{g}(A)$. It is clear from the definition of $\varkappa_{g}(A)$ that $C P_{g}=C$. There is no loss of generality in assuming that $C=0$ and $P_{g}=1$, and that $Q$ is either a continuous, or a discrete finite or properly infinite algebra.

First we assume $\mathbb{Q}$ is continuous. Given $\varepsilon>0$, there is an extreme point $\phi$ of $E_{a}(9)$ such that $\|\phi(A)\|<\varepsilon / 3$, and such that the kernel of the canonical representation $\pi_{\zeta}$ induced by $\phi_{\zeta}$ on the Hilbert space $H_{\zeta}$ is $G+[\zeta]$ for every $\zeta$ in the spectrum $Z$ of $\mathcal{Z}$ (Lemma 2.1). Let $D=A-\phi(A)$ and let $\left\{e_{i} \mid i \in I\right\}$ be a complete orthonormal base of $H_{\zeta}$ so that

$$
\phi_{\zeta}(B)=\left(\pi_{\zeta}(B) e_{1}, e_{1}\right)
$$

for every $B$ in $Q$. Here we are assuming $1 \in I$. For every $S$ in the family $\mathscr{F}(I)$ of all finite subsets of $I-\{1\}$ directed by the inclusion relation, we may find a projection $E(S)$ in $Q$ such that

$$
\pi_{\zeta}(E(S)) e_{1}=e_{1} \text { and } \pi_{\zeta}(E(S)) e_{i}=0 \quad(i \in S)
$$

due to the fact $\pi_{\zeta}$ is irreducible (Lemma 2.1). Because the bounded net $\left\{\pi_{\zeta}(E(S) D E(S)) \mid S \in \mathscr{F}(I)\right\}$ converges to 0 in the strong operator topology on $H_{\zeta}$, there is a set $S$ in $\mathscr{F}(I)$ with

$$
\left\|\pi_{\zeta}(E(S) D E(S)) e_{1}\right\|<\varepsilon / 3 .
$$

The projection $c_{g}(E)$, where $E=E_{\zeta}$ is the spectral projection of $E(S) D^{*} E(S) D E(S)$ in the von Neumann algebra $Q_{E(S)}$ corresponding to the interval $\left[0, \varepsilon^{2} / 3\right]$, is in the ideal $\zeta$; otherwise, the projection $E$ is in the kernel $g+[\zeta]$ of $\pi_{\zeta}$ and a relation

$$
\begin{aligned}
\pi_{\zeta}\left(E(S) D^{*}\right. & E(S) D E(S)) \\
& =\pi_{\zeta}\left((E(S)-E) E(S) D^{*} E(S) D E(S)(E(S)-E)\right) \\
& \geqslant 3^{-1} \varepsilon^{2} \pi_{\zeta}(E(S)-E) \geqslant 3^{-1} \varepsilon^{2} \pi_{\zeta}(E(S))
\end{aligned}
$$

incompatible with the relation (2) arises. Since the map

$$
\xi \rightarrow\left\|\pi_{\xi}(E D E)\right\|=\|E D E(g+[\xi])\|
$$

is continuous on $Z$, we can use the fact that $Z$ is extremally disconnected to find a projection $E$ in $Q$ such that $c_{g}(E)=0$ and such that

$$
\|E D E(9)\|=\operatorname{lub}\{\| E D E(g+[\zeta]) \mid \zeta \in Z\}<\varepsilon / 3
$$

(cf. $[12,3.1])$. If $B$ is an element in 9 with $E B E=B$ and 


$$
\|E D E-B\|<\varepsilon / 3,
$$

and if $F$ is a projection in 9 majorized by $E$ such that

$$
\|B(E-F)\|<\varepsilon / 3,
$$

then we have that $c_{g}(E-F)=0$, or equivalently, that $\operatorname{dim}(E-F)>\operatorname{dim} 9$, and that

$$
\|(E-F) A(E-F)\| \leqslant\|E D E-B\|+\|B(E-F)\|+\|\phi(A)\|<\varepsilon .
$$

Now assume that $Q$ is a type I algebra. There is no loss of generality in the assumption that the commutant $\mathbb{Q}^{\prime}$ of $\mathbb{Q}$ is equal to $\mathscr{Z}$. If $\mathbb{Q}$ is a finite algebra, the assumption that $P_{g}=1$ implies $G=(0)[12,2.3]$. This means that there is a maximal abelian projection $E$ of $\mathscr{Q}$ such that

$$
\|E A E\|=\left\|\tau_{E}(A) E\right\|=\left\|\tau_{E}(A)\right\|<\varepsilon
$$

$[12,4.8]$. If $Q$ is properly infinite type I algebra, let $\left\{E_{i}\right\}$ be a maximal set of nonzero orthogonal abelian projections in $\mathbb{Q}$ such that

$$
\left\|E_{i} A E_{j}\right\| \leqslant 2^{-1} \delta_{i j} \varepsilon
$$

for all $i, j$. Here $\delta_{i j}$ is the Kronecker delta. Setting $E=\sum E_{i}$, we get that

$$
\|E A E\|=\operatorname{lub}\left\|E_{i} A E_{i}\right\| \leqslant \varepsilon / 2 .
$$

We complete the first part of the proof by showing that $c_{g}(E)=0$. On the contrary, if $P=c_{g}(E)$ is nonzero, then the least upper bound $F$ of the range projections of $E P, A E P$, and $A^{*} E P$ is in 9 [7, III, 1, Proposition 2]. If $\phi$ is $E_{a}(\mathcal{G})$ with $\phi(A)=0$, then the map $\psi$ given by

$$
\psi(B)=(P-F) \phi(B)
$$

is a positive $\mathscr{Z}(P-F)$-module homomorphism of the von Neumann algebra $\mathbb{Q}_{(P-F)}$ into its center $\mathscr{Z}(P-F)$ which vanishes on the central ideal $g_{1}$ $=g \cap \mathbb{Q}_{(P-F)}$ of $\mathbb{Q}_{(P-F)}$ and takes $P_{g_{1}}=P-F$ into $P-F$. There is a nonzero abelian projection $G$ in $\mathbb{Q}_{(P-F)}$ such that

$$
\|G A G\| \leqslant \varepsilon / 2
$$

due to the fact that

$$
\psi((P-F) A(P-F))=0
$$

implies that 0 is in $\mathscr{K}_{g_{1}}((P-F) A(P-F))$. Since $G$ is also a nonzero abelian projection in $\mathbb{Q}$ and since 


$$
G E_{i}=G A E_{i}=E_{i} A G=0
$$

for all $i$, the set $\left\{E_{i}\right\}$ is not maximal. This is a contradiction. Hence, we have that $c_{g}(E)=0$.

The converse is already known $[12,4.9]$. Q.E.D.

REMARK. If $C$ is in the essential central range of an operator $A$ with respect to the strong radical in a properly infinite algebra $\mathcal{Q}$, then we have shown elsewhere [11, Proof of Theorem 4] that there is an invertible $B$ in $\mathbb{Q}$ and a projection $E$ equivalent to 1 such that $E B^{-1} A B E=C E$.

The next result answers a question of L. Zsidó [15].

COROllary 2.3. Let $A$ be an element in a properly infinite von Neumann algebra $\mathbb{Q}$ with center $\mathcal{Z}$; then the intersection of $\mathcal{Z}$ with the sets

$$
\delta_{1}=\text { weak closure }\left[\text { convex }\left\{U A U^{*} \mid U \in U(\mathbb{Q})\right\}\right]
$$

and

$$
\delta_{2}=\text { weak closure }\left\{U A U^{*} \mid U \in U(\mathbb{Q})\right\}
$$

coincide. Here $U(\mathbb{Q})$ is the set of unitary operators of $\mathbb{Q}$.

Proof. The ideal $\mathscr{G}_{0}$ of $\mathscr{Q}$ generated by the finite projections of $\mathcal{Q}$ is a central ideal and $\delta_{1} \cap \mathscr{Z}$ is equal to $\mathscr{K}_{g_{0}}(A)[12,4.16]$. If $C$ is in $\mathcal{K}_{g_{0}}(A)$, then there is, for every $n=1,2, \ldots$, a projection $E_{n}$ with $\operatorname{dim} E_{n}>\operatorname{dim} q_{0}$ such that

$$
\left\|E_{n}(C-A) E_{n}\right\|<n^{-1}
$$

(Theorem 2.2). Each projection $E_{n}$ is properly infinite with central support 1 . We may assume that, for each $n$, there is a set $\left\{P_{i} \mid i \in I\right\}$ orthogonal central projections of sum 1 such that each $P_{i}$ is the sum of an infinite set $\left\{E_{i j} \mid j \in J_{i}\right\}$ equivalent orthogonal projections such that $E_{i j}=E_{n} P_{i}$ for some $j$ in $J_{i}$ [7, III, 8 , Theorem 1, Corollary 2 and III, 1, Theorem 1, Corollary 1]. Let $X$ be a finite set of unit vectors in the Hilbert space of $Q$. There is a finite subset $S$ of $I$, and for each $i \in S$, a finite subset $S_{i}$ of $J_{i}$ such that

$$
\left\|\left(1-\sum\left\{P_{i} E_{i j} \mid j \in S_{i}, i \in S\right\}\right) x\right\| \leqslant(2(\|C-A\|+1) n)^{-1}
$$

for all $x$ in $\mathscr{X}$. There is, for every $i$ in $S$, a unitary operator $U_{i}$ in $Q P_{i}$ that carries $F_{i}=E_{n} P_{i}$ onto $G_{i}=\sum\left\{P_{i} E_{i j} \mid j \in S_{i}\right\}$ and $P_{i}-F_{i}$ onto $P_{i}-G_{i}$ because $F_{i}$ $\sim G_{i}$ and $P_{i}-F_{i} \sim P_{i}-G_{i}$. Thus there is a unitary operator $U=U(X, n)$ in $Q$ such that $U P_{i}=U_{i}$ for $i \in S$. Setting $G=\sum\left\{G_{i} \mid i \in S\right\}$ and $P=\sum\left\{P_{i} \mid i\right.$ $\in S\}$, we get that 


$$
\begin{aligned}
|((C-A) U x, U x)| \\
\quad \leqslant 2\|(C-A)\|\|(1-G) x\|+\left|\left((C-A) E_{n} P U x, E_{n} P U x\right)\right| \\
\quad \leqslant n^{-1}+n^{-1}=2 n^{-1}
\end{aligned}
$$

for every $x \in \mathcal{X}$. This proves that $C$ is in the set $\delta_{2} \cap \mathscr{Z}$. Since the relation $\delta_{2} \cap \mathscr{Z} \subset \delta_{1} \cap \mathscr{Z}$ is clear, we see that $\delta_{1} \cap \mathscr{Z}=\delta_{2} \cap \mathscr{Z}$. Q.E.D.

We now prove a stronger version of Theorem 2.2 that will be needed to characterize selfadjoint commutators. For this we need to study the spectral resolution of normal operators in a von Neumann algebra.

Definition 2.4. Let $\Omega$ be the spectrum of the abelian von Neumann algebra $\mathscr{B}$ generated by the center $\mathcal{Z}$ of a von Neumann algebra $\mathcal{Q}$ and a selfadjoint element $A$ in $\mathbb{Q}$; for every element $C$ in the set $\mathscr{Z}_{h}$ of selfadjoint elements of $\mathscr{Z}$, let $E_{A}(C)$ denote the projection in $\mathscr{B}$ whose Gelfand transform is the characteristic function of the open and closed subset closure $\left\{\omega \in \Omega \mid A^{\wedge}(\omega)\right.$ $\left.<C^{\wedge}(\omega)\right\}$ of $\Omega$.

We notice that $\left\{E_{A}(\lambda) \mid-\infty<\lambda<\infty\right\}$ is the spectral resolution of $A$.

Proposition 2.5. Let $A$ be a selfadjoint operator in the von Neumann algebra $Q$ with center $\mathcal{Z}$ and let $G$ be a central ideal of $\mathcal{Q}$. The map

$$
C \rightarrow E_{A}(C)=E(C)
$$

of $\mathscr{Z}_{h}$ into the lattice of projections of the von Neumann algebra $\mathfrak{B}$ generated by $\mathcal{Z}$ and $A$ satisfies the following properties:

(i) $P E(C)=P E(C P)$ for every $C$ in $Z_{h}$ and every projection $P$ in $\mathcal{Z}$;

(ii) $E$ (lub $X)=\operatorname{lub}\{E(C) \mid C \in X\}$ for every subset $\mathcal{X}$ of $\mathscr{I}_{h}$ that is bounded above;

(iii) $(A-C)\left(\right.$ glb $\left.\left\{E\left(C^{\prime}\right) \mid C^{\prime}>C\right\}-E(C)\right)=0$ for every $C$ in $\mathscr{I}_{h}$; and

(iv) $c_{g}(E(C+D)-E(C-D))=0$ for every $D>0$ in $\mathscr{E}_{h}$ if and only if $C \in \mathbb{Z}-\operatorname{Sp}_{g} A$.

Here $C>D$ means that the set $\left\{\omega \in \Omega \mid C^{\wedge}(\omega)>D^{\wedge}(\omega)\right\}$ is dense in $\Omega$.

Proof. (i) Clear.

(ii) This is clear if $\mathscr{X}$ is a monotonely increasing net in $\mathscr{L}$. In general, for any finite subset $C_{1}, \ldots, C_{n}$ of $\mathscr{X}$ there are projections $P_{1}, \ldots, P_{n}$ in $\mathscr{Z}$ of sum 1 such that lub $C_{i}=\sum P_{i} C_{i}$. Using part (i) and the fact that $E(C) \leqslant E(D)$ for $C \leqslant D$ in $\mathscr{E}_{h}$, we have that

$$
\operatorname{lub} E\left(C_{i}\right) \leqslant E\left(\operatorname{lub} C_{i}\right)=\sum P_{i} E\left(C_{i}\right) \leqslant \operatorname{lub} E\left(C_{i}\right)
$$

This means that 


$$
\begin{aligned}
E(\operatorname{lub} \mathcal{X}) & =E\left(\text { lub }\left\{\text { lub } C_{i} \mid\left\{C_{i}\right\} \text { is a finite subset of } \mathcal{X}\right\}\right) \\
& =\operatorname{lub}\left\{E\left(\operatorname{lub} C_{i}\right) \mid\left\{C_{i}\right\} \text { is a finite subset of } \mathcal{X}\right\} \\
& =\operatorname{lub}\{E(C) \mid C \in \mathcal{X}\} .
\end{aligned}
$$

(iii) Let $C^{\prime}=C+n^{-1}$. Then we have that

$$
0 \leqslant(A-C)\left(E\left(C^{\prime}\right)-E(C)\right) \leqslant n^{-1} .
$$

Thus we have

$$
(A-C) \operatorname{glb}\left\{E\left(C^{\prime}\right)-E(C) \mid C^{\prime}>C\right\}=0 .
$$

(iv) Let $C$ be in $\mathscr{Z}-\mathrm{Sp}_{g} A$ and let $\left\{P_{n}\right\}$ be a sequence of orthogonal projections in $\mathcal{Z}$ of sum 1 such that $D P_{n} \geqslant n^{-1} P_{n}$ for every $n=1,2, \ldots$ Because

$$
P(E(C+D)-E(C-D)) \geqslant P\left(E\left(P\left(C+n^{-1}\right)\right)-E\left(P\left(C-n^{-1}\right)\right)\right)
$$

for every central projection $P$ majorized by $P_{n}$ and because $C P$ is in $\mathscr{Z} P-\mathrm{Sp}_{g P} A P[12,3.10]$, it is sufficient to show that the assumption that $\left(E\left(C+n^{-1}\right)-E\left(C-n^{-1}\right)\right)=E$ is in 9 leads to a contradiction. In fact, we have that $(C-A)(1-E)$ is invertible in $B(1-E)$ since $(C-A)^{\wedge}(\omega) \neq 0$ for every $\omega$ in $\Omega$ with $(1-E)^{\wedge}(\omega)=1$. If $B$ is the inverse of $(C-A)(1-E)$ in $B(1-E)$, then $B$ is the inverse of $C-A$ modulo 9 . This contradicts the definition of the essential central spectrum. Thus, we have that $c_{g}(E)=0$.

Conversely, let $C$ in $\mathscr{E}_{h}$ be such that $c_{g}(E(C+D)-E(C-D))=0$ for every $D$ in $\mathscr{Q}_{h}$ with $D>0$. For every $\zeta$ in the spectrum of $\mathscr{L}$ and every $n=1,2, \ldots$, the projection $E\left(C+n^{-1}\right)-E\left(C-n^{-1}\right)$ is not in $9+[\zeta][12$, 3.1] and

$$
\left\|\left(C^{\wedge}(\zeta)-A\right)\left(E\left(C+n^{-1}\right)-E\left(C-n^{-1}\right)\right)(g+[\zeta])\right\| \leqslant n^{-1} .
$$

Hence, each $C^{\wedge}(\zeta)$ is in the spectrum of the canonical image of $A$ in $\mathbb{Q} /(G+[\zeta])$. Q.E.D.

COROLlary 2.6. Let $A$ be a normal element in a von Neumann algebra $\mathfrak{Q}$ with center $\mathcal{Z}$ and let $A_{1}$ (resp. $\left.A_{2}\right)$ be $2^{-1}\left(A+A^{*}\right)\left(\right.$ resp. $\left.(2 i)^{-1}\left(A-A^{*}\right)\right)$. Then an element $C$ in $\mathcal{Z}$ is in the essential central spectrum of $A$ modulo the central ideal $g$ if and only if

$$
c_{g}\left(\left(E_{A_{1}}\left(C_{1}+D_{1}\right)-E_{A_{1}}\left(C_{1}-D_{1}\right)\right)\left(E_{A_{2}}\left(C_{2}+D_{2}\right)-E_{A_{2}}\left(C_{2}-D_{2}\right)\right)\right)=0
$$

for every $D_{1}, D_{2}>0$ in 2 . Here

$$
C_{1}=2^{-1}\left(C+C^{*}\right) \text { and } C_{2}=(2 i)^{-1}\left(C-C^{*}\right) \text {. }
$$

The next result is needed in $\$ 3$. 
COROLlaRy 2.7. If $A$ is a selfadjoint operator in a type $\mathrm{II}_{1}$ von Neumann algebra $Q$, then there are two equivalent orthogonal projections of sum 1 that commute with $A$.

Proof. The set $\mathcal{X}$ of all selfadjoint elements $C$ in the center $\mathscr{Z}$ of $\mathscr{Q}$ such that the value of the canonical operator-valued trace $\Phi$ of $\mathcal{Q}$ (cf. [7, III, 4, Definition 2]) does not exceed $\frac{1}{2}$ at the point $E(C)=E_{A}(C)$ is a bounded monotonely increasing net in $\mathscr{Z}$ (Proposition 2.5(i) and (ii)). We get an element $C_{0}=$ lub $\mathcal{X}$ such that $\Phi\left(E\left(C_{0}\right)\right) \leqslant \frac{1}{2}$ and such that $\Phi(E(C))>\frac{1}{2}$ for every $C>C_{0}$ (Proposition 2.5(ii)). There is a projection $E$ majorized by

$$
F=\operatorname{glb}\left\{E(C)-E\left(C_{0}\right) \mid C>C_{0}\right\}
$$

such that

$$
\Phi(E)=\frac{1}{2}-\Phi\left(E\left(C_{0}\right)\right)
$$

because $\mathbb{Q}$ is a continuous algebra. Since $E$ commutes with $A$ due to the fact $A F=C_{0} F$ (Proposition 2.5(iii)), the projections $E\left(C_{0}\right)+E$ and its orthogonal complement are equivalent orthogonal projections that commute with $A$. Q.E.D.

The next two lemmas are phrased in sufficient generality so that they will be applicable to the matrix decomposition in $\$ 3$.

LEMMA 2.8. Let $E_{i}(i=1,2)$ be projections of central support 1 in the continuous von Neumann algebra $\mathbb{Q}$ and let $\phi$ be a $\sigma$-weakly continuous state of $\mathbb{Q}^{\sim}$ such that $\phi\left(E_{1}\right) \geqslant \alpha>0$. Then for any $\beta$ in the interval $] 0, \alpha$ [ and any $A$ in $\mathbb{Q}$, there are projections $F_{i}(i=1,2)$ of central support 1 majorized by the $E_{i}$ respectively such that $\phi\left(F_{1}\right) \geqslant \beta$ and $F_{2} A F_{1}=0$.

Proof. Let $\mathcal{Z}$ be the center of $\mathcal{Q}$. Given any $P$ in $(\mathscr{Z})$ it is sufficient to show that there is a $Q$ in $(\mathscr{P})$ and projections $F_{i}$ of central support $Q$ majorized by the respective $E_{i}$ such that $\phi\left(F_{1}\right) \geqslant \beta Q$ and $F_{2} A F_{1}=0$. There is no loss of generality in the assumption that $P=1$.

We may assume that $E_{2} A E_{1}$ is not 0 . Let $U$ be a partial isometry and $B$ a positive operator in $\mathbb{Q}$ so that $U B$ is the polar decomposition of $E_{2} A E_{1}$. We may assume that the central support of the range projection $F$ of $B$ is 1 and that the central support $P$ of

$$
E=\operatorname{glb}\left\{E_{B}\left(C^{\prime}\right)-E_{B}(C) \mid C^{\prime}>C\right\},
$$

where $C$ is in $\mathscr{Z} F-\operatorname{Sp} B$ in $\mathbb{Q}_{F}$, is either 0 or 1 . On the one hand, if $P=0$, then the strong limit of the monotonely decreasing sequence

$$
G_{n}=E_{B}\left(C+n^{-1}\right)-E_{B}\left(C-n^{-1}\right) \quad(n=1,2, \ldots)
$$


is 0 and so we can find a number $n$ and a $Q$ in (I) such that

$$
Q \phi\left(G_{n}\right) \leqslant(\alpha-\beta) Q,
$$

and such that $F Q-G_{n} Q$ has central support $Q$. But the projection $G_{n} Q$ also has central support $Q$ (Proposition 2.5(iv)). Hence the projections

$$
F_{1}=Q\left(E_{1}-G_{n}\right) \text { and } F_{2}=U G_{n} U^{*} Q
$$

are projections of central support $Q$ such that $\phi\left(F_{1}\right) \geqslant \beta Q$ and $F_{2} A F_{1}=0$. On the other hand, if $P=1$, then we may write $E$ as the sum of an infinite sequence $\left\{G_{n}^{\prime}\right\}$ of orthogonal projections of central support 1 which commutes

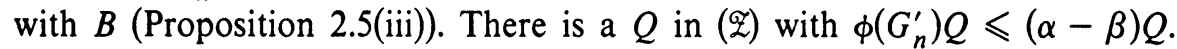
Setting $F_{1}=\left(E_{1}-G_{n}^{\prime}\right) Q$ and $F_{2}=U G_{n}^{\prime} U^{*}$, we get two projections with $\phi\left(F_{1}\right) \geqslant \beta Q$ and $F_{2} A F_{1}=0$. Q.E.D.

LEMMA 2.9. For any finite subset $\mathfrak{X}$ of a properly infinite von Neumann algebra $\mathcal{Q}$ and any sequence $\left\{E_{n}\right\}$ of properly infinite projections of central support 1 , there is a sequence $\left\{E_{n}^{\prime}\right\}$ of equivalent projections of central support 1 such that $E_{m}^{\prime} \leqslant E_{m}$ and $E_{n}^{\prime} A E_{m}^{\prime}=0$ for every $1 \leqslant m<n<\infty$ and $A$ in $\mathcal{X}_{\text {. }}$

Proof. We may assume that $\mathcal{Q}$ is either purely infinite or semifinite. First assume $\mathbb{Q}$ is purely infinite. By passing to a direct summand of $\mathscr{Q}$ and to subprojections of the $E_{n}$ if necessary, we may assume that each $E_{n}$ is $\sigma$-finite [7, III, 1, Lemma 7]. Since the least upper bound of the $E_{n}$ is $\sigma$-finite, we may assume $\mathfrak{Q}$ is $\sigma$-finite by reducing to $\mathbb{Q}_{E}$ if necessary. Applying Lemma 2.7 recursively, we may find projections

$$
\left\{E_{n m} \mid m \geqslant n\right\} \quad(n=1,2, \ldots)
$$

in $\mathscr{Q}$ and corresponding maximal abelian projections $\left\{F_{n}\right\}$ in the commutant of the center $\mathscr{Z}$ of $\mathscr{Q}$ such that

$$
\begin{array}{ll}
E_{n m} \leqslant E_{n p} \leqslant E_{n} & \text { for } n \leqslant p \leqslant m ; \\
E_{n n} A E_{m n}=0 & \text { for } 1 \leqslant m<n \text { and } A \text { in } \chi
\end{array}
$$

and

$$
\tau_{n}\left(E_{n n+k}\right) \geqslant 2-\sum\left\{3^{-j} \mid 0 \leqslant j \leqslant k\right\} \text { for } k=0,1, \ldots
$$

Here $\tau_{n}=\tau_{F_{n}}$. Setting $E_{n}^{\prime}=\mathrm{glb}_{m} E_{n m}$, we get that

$$
\tau_{n}\left(E_{n}^{\prime}\right)=\operatorname{glb} \tau_{n}\left(E_{n m}\right) \geqslant 2^{-1}
$$

so that $E_{n}^{\prime}$ is equivalent to 1 [7, III, 8, Theorem 1, Corollary 5] and that $E_{n}^{\prime} A E_{m}^{\prime}=0$ for $1 \leqslant m<n<\infty$ and $A$ in $\mathcal{X}$. 
Now let $\mathfrak{Q}$ be semifinite. Suppose we have found equivalent finite projections $E_{1}^{\prime}, E_{2}^{\prime}, \ldots, E_{n}^{\prime}$ of central support 1 such that $E_{m}^{\prime} \leqslant E_{m}$ for $m=1,2, \ldots, n$ and $E_{p}^{\prime} A E_{m}^{\prime}=0$ for $1 \leqslant m<p \leqslant n$ and $A$ in $\mathcal{X}$. The least upper bound $F$ of the range projections of $E_{n+1} A E_{m}^{\prime}$ for $1 \leqslant m \leqslant n$ and $A$ in $\mathcal{X}$ is a finite projection, and so there is a projection $E_{n+1}^{\prime}$ with $E_{n}^{\prime} \sim E_{n+1}^{\prime} \leqslant E_{n+1}-F$. By induction the required sequence $\left\{E_{n}^{\prime}\right\}$ exists. Q.E.D.

We now obtain the desired extension of Theorem 2.2.

THEOREM 2.10. Let $\mathbb{Q}$ be a von Neumann algebra with center $\mathscr{Z}$, and let $g=g_{Q}(G)$ be a central ideal of $\mathbb{Q}$ in canonical form. Suppose that $\mathbb{Q}(1-Q)$ is a continuous von Neumann algebra. Let 0 be in the essential central spectrum modulo $g$ of an element $A$ in $Q_{0}$. For any sequence $\left\{\alpha_{n}\right\}$ of positive numbers and any finite subset $\mathcal{X}$ of $\mathbb{Q}$, there is a sequence $\left\{E_{n}\right\}$ of projections in $\mathbb{Q}$ such that

(1) $\operatorname{dim} E_{n}>\operatorname{dim} 9$;

(2) $E_{n} B E_{m}=0$ for all $1 \leqslant m<n<\infty$ and $B$ in $\mathscr{X}$; and

(3) $\left\|E_{n} A E_{n}\right\| \leqslant \alpha_{n}$ for all $n$.

In particular, if 1 is in $\mathcal{X}$, then the projections $\left\{E_{n}\right\}$ are orthogonal.

REMARK. For the applications in $\$ 3$ it is important to notice that the strong radical $g$ of a properly infinite algebra $\mathbb{Q}$ satisfies the hypothesis of Theorem 2.10. We recall, in fact, that $Q Q$ is the weak closure of the central ideal $g_{Q}(G)$ (cf. Introduction) and so $Q(1-Q)$ is of type III or (0) if $g_{Q}(G)=g$ because $g$ contains all finite projections.

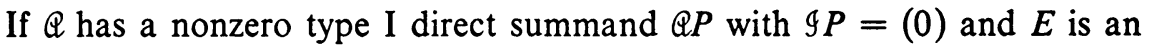
abelian projection of central support $P$, there is no orthogonal sequence $\left\{E_{n}\right\}$ of projections of central support $P$ that satisfies (3) for $\alpha_{n}=2^{-1}(n=1$, $2, \ldots)$ and $A=1-E$.

ProOF. We may assume that $P_{g}=1$, and by passing to direct summands, that $Q$ is continuous and $G=(0)$ or that the support of $G$ is 1 .

If $\mathbb{Q}$ is continuous and $G=(0)$, then there is a sequence $\left\{F_{n}\right\}$ of projections with central support 1 such that $\left\|F_{n} A F_{n}\right\| \leqslant \alpha_{n}$. The desired sequence $\left\{E_{n}\right\}$ may be constructed in a recursive fashion from Lemma 2.8 (cf. Proof, Lemma 2.9).

Now suppose the support of $g$ is 1 . We also suppose that $\mathcal{X}$ contains the identity 1 . There is a sequence $\left\{F_{n}\right\}$ of projections in $\mathcal{Q}$ with $\operatorname{dim} F_{n}>\operatorname{dim} g$ and $\left\|F_{n} A F_{n}\right\| \leqslant \alpha_{n}$ for every $n=1,2, \ldots$ (Theorem 2.2). The projections $F_{n}$ must be properly infinite projections of central support 1 since every finite projection is in $\mathscr{G}(12,2.2)$. Let $\delta$ be the set of all families

$$
S=\left\{E_{n i} \mid(n, i) \in N \times I\right\}
$$

of nonzero projections in $\mathscr{Q}$ with the following properties

(i) $E_{m i} \sim E_{n i}$ for all $m, n$ in $N$ and $i \in I$; 
(ii) $E_{n j} B E_{m i}=0$ for all distinct pairs $(m, i),(n, j)$ in $N \times I$ and $B$ in Y $=\mathscr{X} \cup \mathcal{X}^{*} \cup\left\{A, A^{*}\right\}$; and

(iii) $\left\|E_{n i} A E_{n i}\right\| \leqslant \alpha_{n}$ for $n$ in $N$ and $i \in I$.

Here $\mathcal{X}^{*}=\left\{B^{*} \mid B \in \mathcal{X}\right\}$ and $N$ is the set of natural numbers. The set $\delta$ is nonvoid, and in fact, for every nonzero central projection $P$, there is a sequence $\left\{E_{n}^{\prime}\right\}$ of equivalent orthogonal projections of central support $P$ such that $E_{n}^{\prime} \leqslant F_{n}$ and $E_{n}^{\prime} B E_{m}^{\prime}=0$ for all $1 \leqslant m<n<\infty$ and $B$ in $\mathcal{Y}$ (Lemma 2.9). By Zorn's lemma we may find a maximal set $S=\left\{E_{n i} \mid(n, i) \in N \times I\right\}$ in the partial ordering on $\delta$ given by the relation

$$
\left\{E_{n i}^{\prime} \mid(n, i) \in N \times I^{\prime}\right\}<\left\{E_{n i}^{\prime \prime} \mid(n, i) \in N \times I^{\prime \prime}\right\}
$$

if and only if $I^{\prime} \subset I^{\prime \prime}$ and $E_{n i}^{\prime}=E_{n i}^{\prime \prime}$ for $i \in I^{\prime}$. For convenience, let $k$ be a point not in $I$. Let $\left\{N_{m} \mid m \in N\right\}$ be a partition of the natural numbers into countably many disjoint infinite sets such that the smallest number in the set $N_{m}$ is not smaller than $m$, and let

$$
E_{m}=\sum\left\{E_{n i} \mid(n, i) \in N_{m} \times I\right\}
$$

for $m=1,2, \ldots$ We show that each projection $E_{n}$ has dimension greater than that of $g$ by showing $c_{g}\left(E_{n}\right)=0$. First we notice that each projection $E_{n}^{\prime}=\sum\left\{E_{n i} \mid i \in I\right\}$ has central support $P$ equal to 1 ; otherwise, there would be a sequence $\left\{E_{n k}\right\}$ of central support $1-P$ such that

$$
\left\{E_{n i} \mid(n, i) \in N \times(I \cup\{k\})\right\}
$$

lies in $\delta$. Since the projections $E_{n}^{\prime}$ are equivalent, the projections $E_{n}$ are equivalent, properly infinite, orthogonal projections of central support 1 [7, III 2, Proposition 10]. We show that $c_{g}\left(E_{1}\right)=P$ is zero. On the contrary, assume $P$ is nonzero. We may write $E_{1}$ as the sum of an infinite sequence $\left\{G_{n}\right\}$ of orthogonal projections each equivalent to $E_{1}$ and thus to each $E_{m}$. Because

$$
P \sum E_{n} \prec P \sum G_{n}=P E_{1},
$$

we get that $P \sum E_{n}$ is in 9 . The least upper bound $E$ of the range supports of the finite set of elements $\left\{P B \sum E_{n} \mid B \in \mathcal{Y}\right\}$ is also a projection in 9 . This means that the algebra $C=\mathscr{Q}_{(P-E)}$ is properly infinite and that 0 is in the essential range of the element $C=(P-E) A(P-E)$ in $C$ modulo the central ideal $g^{\prime}=g \cap \mathcal{C}$ of $\mathcal{C}$. Indeed, if $\phi$ is a positive $\mathscr{L}$-module homomorphism of the algebra $\mathscr{Q}$ into $\mathscr{Z}$ with $\phi(1)=1, \phi(A)=0$ and $\phi(\mathcal{G})=(0)$, then the function $\psi$ on $C$ given by

$$
\psi(D)=(P-E) \phi(D)
$$


is a positive module homomorphism of $C$ into its center $\mathscr{Z}(P-E)$ with $\psi(P-E)=P-E, \psi(C)=0$, and $\psi\left(g^{\prime}\right)=(0)$. There is a sequence $\left\{F_{n}^{\prime}\right\}$ of

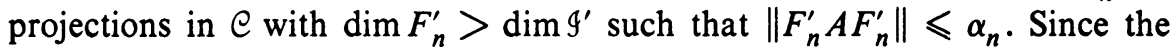
weak closure of $g^{\prime}$ is $C$ and since the orthogonal complement of the largest central projection in $\mathcal{C}$ that is in $\mathcal{G}^{\prime}$ is 0 , the projections $F_{n}^{\prime}$ are properly infinite and of central support $P-E$ in $C$. There are equivalent projections $\left\{E_{n k} \mid n \in N\right\}$ of central support 1 such that $E_{m k} \leqslant F_{m}^{\prime}$ and $E_{n k} B E_{m k}=0$ for $1 \leqslant m<n<\infty$ and $B$ in the set $\{(P-E) D(P-E) \mid D \in \mathcal{Y}\}$. Then the set

$$
\left\{E_{n i} \mid(n, i) \in N \times(I \cup\{k\})\right\}
$$

is in $\delta$. This is a contradiction. Hence, we have that $c_{g}\left(E_{1}\right)=0$ and that $\operatorname{dim} E_{n}>\operatorname{dim} 9$. Thus $\left\{E_{n}\right\}$ is a sequence of projections satisyfing property (1). We show that $\left\{E_{n}\right\}$ also satisfies (2) and (3). In fact, for $1 \leqslant m<n<\infty$ and $B$ in $\mathcal{X}$, we have that

$$
\left\|E_{n} B E_{m}\right\|=\left\|\sum\left\{E_{p i} B E_{q j} \mid(p, q, i, j) \in N_{n} \times N_{m} \times I \times I\right\}\right\|=0
$$

from (ii), and that

$$
\begin{aligned}
\left\|E_{m} A E_{m}\right\| & =\left\|\sum\left\{E_{p i} A e_{q j} \mid(p, q, i, j) \in N_{m} \times N_{m} \times I \times I\right\}\right\| \\
& =\left\|\sum\left\{E_{p i} A E_{p i} \mid(p, i) \in N_{m} \times I\right\}\right\| \\
& =\operatorname{lub}\left\{\left\|E_{p i} A E_{p i}\right\| \mid(p, i) \in N_{m} \times I\right\} \leqslant \alpha_{m}
\end{aligned}
$$

from (ii) and (iii). Q.E.D.

We now examine the relation of the essential central spectrum and essential central range.

COROLlary 2.11. Let $Q$ be a von Neumann algebra and let $g=g_{Q}(G)$ be a central ideal of $\mathbb{Q}$ in canonical form. Suppose that $\mathbb{Q}(1-Q)$ is a continuous von Neumann algebra. Then an element $C$ in $\mathcal{Z}$ is in the essential central range of an element $A$ in $Q$ modulo the central ideal $G$ if and only if there is a projection $E$ in $Q$ with $\operatorname{dim} E>\operatorname{dim} 9$ such that $C E$ is in the essential central spectrum of $E A E$ in $\mathbb{Q}_{E}$ modulo the central ideal $9 \cap E Q E$.

Proof. There is no loss of generality in that assumption that $P_{g}=1$ and $C=0$. If 0 is in $\mathscr{K}_{g}(A)$, there is a sequence $\left\{E_{n}\right\}$ of projections in $\mathbb{Q}$ with $\operatorname{dim} E_{n}>\operatorname{dim} 9,\left\|E_{m} A E_{m}\right\| \leqslant m^{-1}$, and $E_{n} B E_{m}=0$ for $1 \leqslant m<n<\infty$ and $B$ in the set $\left\{1, A, A^{*}\right\}$ (Theorem 2.10). Let $E$ be the projection $E=\sum E_{n}$. Each $E_{m}$ has dimension greater than that of $\mathscr{G}^{\prime}=\mathscr{G} \cap E Q E$ since 


$$
c_{g^{\prime}}\left(E_{m}\right)=c_{g}\left(E_{m}\right) E=0 .
$$

Since

$$
\lim \left\|E A E E_{m}\right\|=\lim \left\|E_{m} A E_{m}\right\|=0,
$$

we get that 0 is in $\mathscr{L} E-\mathrm{Sp}_{g^{\prime}} E A E$.

Conversely, let $E$ be a projection in $\mathscr{Q}$ with $\operatorname{dim} E>\operatorname{dim} 9$ such that 0 is in the essential central spectrum of $E A E$ modulo $q^{\prime}=q \cap \mathbb{Q}_{E}$. For every $m=1,2, \ldots$, there is an $E_{m}$ in $\mathbb{Q}_{E}$ with $\operatorname{dim} E_{m}>\operatorname{dim} q^{\prime}$ such that $\left\|E_{m} E A E E_{m}\right\| \leqslant m^{-1}$ (cf. [12, 3.16]). Because $E$ has central support 1, we get that $\operatorname{dim} E_{m}>\operatorname{dim} 9$ from relation (4). We conclude from Theorem 2.2 that $\mathscr{K}_{g}(A)$ contains 0 . Q.E.D.

3. Selfadjoint commutators. In this section the selfadjoint commutators in properly infinite von Neumann algebras are characterized as those elements that contain 0 in their essential central range modulo the strong radical. A matrix form similar to that used for bounded operators on a separable space in [1] is found for such operators.

Definition 3.1. A sequence $\left\{E_{n} \mid 1 \leqslant n<m\right\}$ (where $m$ is a finite or countable cardinal) of orthogonal projections in a properly infinite von Neumann algebra is said to be a matrix base if $1 \sim E_{1} \sim E_{2} \sim \cdots$ and $\sum E_{n}=1$.

Let $\left\{E_{n} \mid 1 \leqslant n \leqslant m\right\}$ be a matrix base for the properly infinite algebra $\mathbb{Q}$ and let $U_{n}$ be partial isometries of $\mathscr{Q}$ with domain support $E_{n}$ and range support 1 . Then each element $A$ in $\mathscr{Q}$ can be written as

$$
A=\sum E_{n} A E_{m}=\sum U_{n}^{*} \cdot U_{n} A U_{m}^{*} \cdot U_{m} .
$$

The $m \times m$-matrix $\left(U_{n} A U_{m}^{*}\right)$ is said to be a matrix with regard to the base $\left\{E_{n}\right\}$. The matrix depends on the choice of the partial isometries; however, in the sequel the choice of the matrix base is critical and the choice of the partial isometries is immaterial.

We now begin a series of matrix reductions. The first extends the result in Corollary 2.7.

LEMma 3.2. For every selfadjoint operator $A$ in a properly infinite von Neumann algebra \&, there is a matrix base $\left\{E_{i} \mid 1 \leqslant i \leqslant 2\right\}$ for $\mathbb{Q}$ that commutes with $A$.

Proof. Let $g$ be the strong radical of $\mathcal{Q}$, let $\mathcal{Z}$ be the center of $\mathscr{Q}$, and let $C_{u}$ and $C_{l}$ be the least upper bound and greatest lower bound respectively of $\mathscr{Z}-\mathrm{Sp}_{g} A[12,3.11]$. Since there is a projection $P$ in $\mathscr{Z}$ with $P C_{u}>P C_{l}$ and $(1-P) C_{u}=(1-P) C_{l}$, we may assume that either $C_{u}>C_{l}$ or that $C_{u}$ $=C_{l}$. 
If $C_{u}>C_{l}$, then the projections $E=E_{A}\left(C_{u}+D\right)-E_{A}\left(C_{u}-D\right)$ and $1-E$ are equivalent to 1 whenever $0<D \leqslant 2^{-1}\left(C_{u}-C_{l}\right)$ due to Proposition 2.5(iv). Hence, we may assume $C_{u}=C=C_{l}$.

Let $E$ denote the range projection of the selfadjoint operator $C-A$ in $g$; then the projection $c_{g}(E)(1-E)$, which annihilates $C-A$, is equivalent to $c(E)=c_{g}(E)$ and can be written as the sum of two orthogonal projections $E_{1}$, $E_{2}$ both equivalent to $c(E)$ [7, III, 8, Theorem 1, Corollary 2]. The projections $E_{1}+c(E) E$ and $E_{2}$ form a matrix base for $\mathbb{Q} c(E)$ that commute with $c(E)(C-A)$ and thus with $c(E) A$. So we may assume that $c(E)=0$, or equivalently, that $E \sim 1$.

It is sufficient to show that given any selfadjoint operator $A$ in $g$ with range support equivalent to 1 , there are equivalent orthogonal projections of sum 1 that commute with $A$. We prove first that there are at least two nonzero equivalent orthogonal projections majorized by the range support $E$ of $A$ that commute with $A$. Assume for the present that $E=1$. Let $C_{1}, C_{2}$ be in $\mathscr{Z}$ with $C_{1}>C_{2}>0$ and let $F\left(C_{1}, C_{2}\right)$ be the projection in $g$ given by

$$
F\left(C_{1}, C_{2}\right)=\left(E\left(C_{1}\right)-E\left(C_{2}\right)\right)+\left(E\left(-C_{2}\right)-E\left(-C_{1}\right)\right)
$$

where $E\left(C_{2}\right)=E_{A}\left(C_{2}\right)$ (cf. Definition 2.4). By decomposing into the product of two algebras, we may suppose that either there is a $C_{1}$ in $\mathscr{L}$ with

$$
\|A\|+1=C_{0}>C_{1}>0
$$

such that $F\left(C_{0}, C_{1}\right)$ is properly infinite of central support 1 or that $F\left(C_{0}, C_{1}\right)$ is finite or zero for every $C_{0}>C_{1}>0$ (Proposition 2.5(i)).

First assume $F\left(C_{0}, C_{1}\right)$ is properly infinite of central support 1. We show that there is a $C_{2}$ in $\mathscr{Z}$ with $C_{1}>C_{2}>0$ such that

$$
F\left(C_{1}, C_{2}\right)>F\left(C_{0}, C_{1}\right) \text {. }
$$

In fact, we show more: for any $C, C^{\prime}$ in $\mathscr{Z}$ with $C>C^{\prime}>0$ so that $F\left(C, C^{\prime}\right)$ is properly infinite of central support 1 , there is a $C^{\prime \prime}$ in $\mathcal{Z}$ with $C^{\prime}>C^{\prime \prime}>0$ such that

$$
F\left(C^{\prime}, C^{\prime \prime}\right) \succ F\left(C, C^{\prime}\right)
$$

Since we have that

$$
P E(D)=P E(P D)
$$

for every selfadjoint $D$ in $\mathscr{Z}$ (Proposition 2.5(i)), it is sufficient to show that for any $P$ in ( $(2)$ there is a $Q$ in $\left(\mathscr{Z} P\right.$ ) and a $C^{\prime \prime}$ in $Z Q$ with $C^{\prime} Q>C^{\prime \prime} Q>0$ and

$$
Q F\left(C^{\prime} Q, C^{\prime \prime} Q\right)>Q F\left(C Q, C^{\prime} Q\right) .
$$


There is no loss of generality in the assumption that $P=1$. We show that the relation

$$
F\left(C, C^{\prime}\right)>F\left(C^{\prime}, C^{\prime \prime}\right)
$$

for all $C^{\prime \prime}$ with $C^{\prime}>C^{\prime \prime}>0$ leads to a contradiction. In fact let $\left\{F_{n}\right\}$ be a sequence of orthogonal projections of sum $F\left(C, C^{\prime}\right)$ such that $F_{n} \sim F\left(C, C^{\prime}\right)$ for every $n[7$, III, 8 , Theorem 1 , Corollary 2]. We have that

$$
1 \sim 1-F\left(C_{0}, C^{\prime}\right)=\sum F\left(m^{-1} C^{\prime},(m+1)^{-1} C^{\prime}\right) \prec \sum F_{n} \leqslant F\left(C, C^{\prime}\right)
$$

because the range projection of $A$ given by $1-E(0+)+E(0)$ is equal to 1 . This is a contradiction due to the fact $F\left(C, C^{\prime}\right)$ is in $g$. So there is a $C^{\prime \prime}$ in $\mathscr{Z}$ with $C^{\prime}>C^{\prime \prime}>0$ such that

$$
F\left(C^{\prime}, C^{\prime \prime}\right)>F\left(C, C^{\prime}\right)
$$

Noting that $F\left(C^{\prime}, C^{\prime \prime}\right)$ in the previous expression is properly infinite of central support 1 , we may find a strictly decreasing sequence $\left\{C_{n}\right\}$ in $\mathscr{L}^{+}$such that $F\left(C_{0}, C_{1}\right) \prec F\left(C_{1}, C_{2}\right) \prec \cdots$. Setting

$$
E_{1}=\sum F\left(C_{2 n}, C_{2 n+1}\right) \text { and } E_{2}=\sum F\left(C_{2 n-1}, C_{2 n}\right),
$$

we obtain two orthogonal projections $E_{1}$ and $E_{2}$ of sum 1 such that

$$
E_{2} \prec E_{1} \prec F\left(C_{0}, C_{1}\right)+E_{2} .
$$

Since $F\left(C_{0}, C_{1}\right)$ is in the strong radical, we get that $E_{1} \sim E_{2} \sim 1$.

Now we assume that $F\left(C_{0}, n^{-1}\right)$ is a finite or zero projection for every $n=1,2, \ldots$. If $Q$ is a continuous algebra, then there are, for each $n=1,2$, ..., two equivalent orthogonal projections of sum $F\left(C_{0}, n^{-1}\right)$ which commute with $A F\left(C_{0}, n^{-1}\right)$ and consequently with $A$ (Corollary 2.7). Since $F\left(C_{0}, n^{-1}\right)$ is nonzero for some $n$ (Proposition 2.5(iii)), we obtain nonzero equivalent orthogonal projections commuting with $A$. Therefore, we may assume that $\mathbb{Q}$ is of type I. There is an $n$ such that $F\left(C_{0}, n^{-1}\right)$ is not an abelian projection; otherwise, the properly infinite projection 1 can be expressed as the least upper bound of a monotonely increasing sequence $\left\{F\left(C_{0}, n^{-1}\right)\right\}$ of abelian projections which is also an abelian projection. This means that there is an $n$ and a

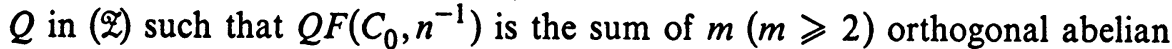
projections of central support $Q$. Because $A F\left(C_{0}, n^{-1}\right) Q$ can be represented as a diagonal $m \times m$ operator matrix over $\mathscr{Z} F\left(C_{0}, n^{-1}\right) Q$ based on matrix units arising from the $m$ equivalent orthogonal abelian projections of sum $F\left(C_{0}, n^{-1}\right) Q$ [6], we may find two nonzero orthogonal equivalent projections (actually abelian projections) which commute with $A F\left(C_{0}, n^{-1}\right) Q$ and thus with $A$. 
In every case we have found two nonzero equivalent orthogonal projections that commute with $A$. We now remove the assumption that the range projection $E$ is equal to 1 . Let $V$ be an isometry in $Q$ whose range support is $E$. The element $V^{*} A V$ is a selfadjoint element in the strong radical whose range support is 1 . As was just shown, there are nonzero orthogonal equivalent projections $E_{1}$ and $E_{2}$ that commute with $V A V^{*}$. The projections $V E_{1} V^{*}$ and $V E_{2} V^{*}$ are nonzero equivalent orthogonal projections majorized by $E$ that commute with $A$.

We now construct the desired projections by a maximality argument. Let $\left\{F_{i}, G_{i} \mid i \in I\right\}$ be a maximal set of nonzero mutually orthogonal projections majorized by $E$ that commute with $A$ such that $F_{i} \sim G_{i}$ for every $i$. The projections $F=\sum F_{i}$ and $G=\sum G_{i}$ are equivalent orthogonal projections majorized by $E$ that commute with $A$. The projection

$$
E^{\prime}=c(F)(E-(F+G))
$$

is equivalent to $c(F)$ since $c(F)=c(G)$, and the range support $E^{\prime}$ of $A E^{\prime}$ in the strong radical of the properly infinite von Neumann algebra $\mathbb{Q} c(F)$ is equivalent to $c(F)$. This means $c(F)=0$; otherwise, the arguments of the preceding paragraphs can be applied to show that $\left\{F_{i}, G_{i}\right\}$ is not maximal. Thus we have that the projections $F, 1-F$ form a matrix base that commutes with A. Q.E.D.

ReMARK. Combining the results of Corollary 2.7, Lemma 3.2, and [6], we obtain that, for every selfadjoint operator $A$ in a von Neumann algebra with no type $\mathrm{I}_{n}(n$ odd) part, there are two equivalent orthogonal projections of sum 1 commuting with $A$.

The next lemma extends the results of Anderson and Stampfli [2].

LemMa 3.3. Let $A$ be an element in a properly infinite von Neumann algebra $\mathbb{Q}$; then there is a matrix base $E_{1}, E_{2}\left(\right.$ resp. $\left.F_{1}, F_{2}\right)$ of $\&$ such that $F_{1} A E_{2}=F_{2} A E_{1}$ $=0$.

Proof. Let $B$ be a positive selfadjoint operator in $\mathcal{Q}$ and let $U$ be a partial isometry of $Q$ so that $A=U B$ is the polar decomposition of $A$. The projections $c\left(U^{*} U\right)\left(1-U^{*} U\right)$ and $c\left(U^{*} U\right)\left(1-U U^{*}\right)$ may be written as the sum of two equivalent orthogonal projections $E, E^{\prime}$ and $F, F^{\prime}$ respectively. Here $c\left(U^{*} U\right)=c_{g}\left(U^{*} U\right)$ where $g$ is the strong radical of $\mathscr{Q}$. The projections

$$
E_{1}=E+c\left(U^{*} U\right) U^{*} U, \quad E_{2}=E^{\prime}
$$

and

$$
F_{1}=F+c\left(U^{*} U\right) U U^{*}, \quad F_{2}=F^{\prime}
$$

are equivalent orthogonal projections of $\operatorname{sum} c\left(U^{*} U\right)$ respectively with 


$$
F_{1} A E_{2}=F_{2} A E_{1}=0 .
$$

So we may assume that $c\left(U^{*} U\right)=0$. There are equivalent orthogonal projections $E, E^{\prime}$ of sum $U^{*} U$ that commute with $B$ due to the fact that $U^{*} U$ is properly infinite (Lemma 3.2). The projections $U E U^{*}$ and $U E^{\prime} U^{*}$ are orthogonal equivalent projections of sum $U U^{*}$. Setting

$$
E_{1}=E+\left(1-U^{*} U\right), \quad E_{2}=E^{\prime}
$$

and

$$
F_{1}=U E U^{*}+\left(1-U U^{*}\right), \quad F_{2}=U E^{\prime} U^{*},
$$

we get matrix bases that satisfy the desired relations. Q.E.D.

We now are ready to characterize a selfadjoint commutator. We treat the product of $\sigma$-finite type I algebras separately. This case is slightly anomalous and in fact follows from the preceding lemmas via the analysis of $\mathrm{J}$. Anderson [1]. In the sequel let $\alpha_{n}=((n+1) !)^{-1}$ for $n=1,2, \ldots$

THEOREM 3.4. Let $\mathbb{Q}$ be the product of $\sigma$-finite properly infinite type I von Neumann algebras. Then an element $A$ in $\mathbb{Q}$ is a selfadjoint commutator in $\mathbb{Q}$ if and only if 0 is in the essential central range of $A$ modulo the strong radical $g$ of $Q$.

Proof. First let $A$ have the matrix representation

$$
\left(\begin{array}{ll}
* & 0 \\
0 & B
\end{array}\right)
$$

where $B \in \mathcal{G}$. Since $\mathcal{K}\left(B^{*} B+B B^{*}\right)=\{0\}$, we may write $B$ as the infinite matrix operator $B=\left(B_{i j}\right)$ over $\mathcal{Q}$ with $\left\|B_{i j}\right\| \leqslant 2\|B\| \min \left\{\alpha_{i}, \alpha_{j}\right\}$. In fact, by applying Theorem 2.10 to $B^{*} B+B B^{*}$, we may find a sequence $\left\{E_{n}\right\}$ of orthogonal projections in $\mathscr{Q}$ such that $\operatorname{dim} E_{n}>\operatorname{dim} g$ and

$$
\left\|E_{n}\left(B^{*} B+B B^{*}\right) E_{n}\right\| \leqslant 4 \alpha_{n}^{2}\|B\|^{2}
$$

for all $1 \leqslant n<\infty$. Replacing $E_{1}$ by $1-\sum\left\{E_{n} \mid n \geqslant 2\right\}$, we get a matrix base $\left\{E_{n}\right\}$ of $\mathscr{Q}$ such that

$$
\left\|E_{n} B E_{m}\right\| \leqslant 2\|B\| \min \left\{\alpha_{m}, \alpha_{n}\right\}
$$

The calculation of M. David [5, Theorem 3] now shows that $A$ is a selfadjoint commutator.

Now let $A$ be an operator in $\mathscr{Q}$ such that $\Re(A)$ contains 0 . Applying Theorem 2.10, we may find an orthogonal sequence $\left\{F_{n}\right\}$ of maximal abelian projections of $\mathscr{Q}$ of $\operatorname{sum} F$ such that $1-F \sim F \sim 1$ and such that 


$$
\left\|F_{m} A F_{n}\right\| \leqslant n^{-1} \delta_{m n}
$$

for all $m, n=1,2, \ldots$ [7, III, 8, Theorem 1, Corollary 5]. Here $\delta$ is the Kronecker delta. We have that $F A F$ is in $g$ since $G=\sum\left\{F_{n} \mid 1 \leqslant n \leqslant m\right\}$ is in $g$ and

$$
\|F A F(1-G)\|=\operatorname{lub}\left\{\left\|F_{n} A F_{n}\right\| \mid n>m\right\} \leqslant(m+1)^{-1} \text {. }
$$

The 2,2 term of the matrix of $A$ induced by the matrix base $1-F, F$ is in $g$. Using Lemma 3.3, we can follow the analysis of J. Anderson [1] to write $A$ as an $8 \times 8$-matrix over $\mathbb{Q}$ such that each of the four $2 \times 2$ diagonal blocks is of the form (5). Thus $A$ is a selfadjoint commutator.

Conversely, let $A=B C-C B$ with $B=B^{*}$ and $C$ in $Q$. We may assume that 0 is in $\mathscr{Z}-\operatorname{Sp}_{\mathfrak{g}} B$. Given $\varepsilon>0$, there is a projection $E$ in $\mathbb{Q}$ with $\operatorname{dim} E>\operatorname{dim} g$ and with $\|B E\|=\|E B\|<\varepsilon$ (Proposition 2.5(iv)). We have that $\|E A E\| \leqslant 2 \varepsilon\|C\|$, and thus that $0 \in \mathcal{K}_{g}(A)$. Q.E.D.

Now we can restrict our attention to properly infinite algebras with no $\sigma$ finite type I direct summands.

LEMMA 3.5. Let $A$ be a selfadjoint operator in a properly infinite von Neumann algebra $\mathbb{Q}$ with no $\sigma$-finite type I direct summands and let $\left\{E_{n}\right\}$ be a sequence of projections in $\mathbb{Q}$ which commute with $A$; then for every $n=1,2, \ldots$, there are equivalent orthogonal projections $E_{n 1}$ and $E_{n 2}$ commuting with $A$ and majorized by $E_{n}$ such that lub $\left(E_{n}-\left(E_{n 1}+E_{n 2}\right)\right)$ is in the strong radical of $\mathcal{Q}_{\text {. }}$

Proof. We may assume that either $\mathbb{Q}$ is a continuous $\sigma$-finite algebra or that $\mathcal{Q}$ has no $\sigma$-finite direct summand. In case $\mathbb{Q}$ is a continuous $\sigma$-finite algebra, we show that $E_{n}$ may in fact be written as the sum of two equivalent orthogonal projections commuting with $A$. Let $P_{n}$ be the largest central projection in $\mathbb{Q}$ such that $E_{n} P_{n}$ is finite. Then $E_{n} P_{n}$ (resp. $\left.E_{n}\left(1-P_{n}\right)\right)$ may be written as the sum of two equivalent orthogonal projections commuting with $A E_{n} P_{n}$ (resp. $A E_{n}\left(1-P_{n}\right)$ ) due to Corollary 2.7 (resp. Lemma 3.2). So $E_{n}$ may be written as the sum of two equivalent orthogonal projections commuting with $A$.

Now suppose $\mathbb{Q}$ has no $\sigma$-finite direct summands. For every $n=1,2, \ldots$, we may use the reasoning of the preceding paragraph to find two equivalent orthogonal projections $E_{n 1}, E_{n 2}$ commuting with $A$ and majorized by $E_{n}$ so that $E_{n}-\left(E_{n 1}+E_{n 2}\right)$ is a finite projection. To complete the proof it is sufficient to show that the least upper bound $F$ of a monotonely increasing sequence $\left\{F_{n}\right\}$ of finite projections in a von Neumann algebra with no $\sigma$-finite direct summands is in the strong radical [7, III, 2, Proposition 5]. Since the strong radical is a central ideal, and since any direct summand of a finite projection is finite, it is sufficient to show that there is a nonzero central projection $Q$ such that $F Q$ is in the strong radical. Let $x$ be a unit vector in 
the Hilbert space of $Q_{\text {. For each }} n=1,2, \ldots$, there is an orthogonal set $\left\{Q_{i}\right\}$ central projections of sum 1 such that $F_{n} Q_{i}$ is $\sigma$-finite [7, I, 6,Proposition 9(iii)]. There is a finite subset of the $Q_{i}$ of sum $Q_{n}$ such that $\left\|x-Q_{n} x\right\| \leqslant 2^{-n-1}$. Setting $Q$ equal to the greatest lower bound of the monotonely decreasing sequence $\left\{Q_{1} Q_{2} \cdots Q_{n}\right\}$ of central projections, we obtain the required nonzero projection because $\|x-Q x\| \leqslant 2^{-1}$ and because $F Q$ is $\sigma$-finite and consequently in the strong radical. Q.E.D.

If 0 is in the essential central range of $A$ modulo the strong radical of the properly infinite algebra $Q$, the projections $\left\{E_{n}\right\}$ of Theorem 2.10 induce the matrix decomposition $A=\left(C_{i j}\right)$ over $\mathscr{Q}$ with $\left\|C_{m n}\right\| \leqslant \alpha_{n} \delta_{m n}$ for $m, n \geqslant 2$. Here we replace $E_{1}$ of Theorem 2.10 by $1-\sum\left\{E_{n} \mid n \geqslant 2\right\}$, which majorizes $E_{1}$ and is thus equivalent to 1 . We may also choose the $E_{n}$ so that $E_{m} B E_{n}=0$ for $2 \leqslant m<n<\infty$ and $B$ in a preassigned finite subset of $Q$. We now obtain a second matrix form for $A$.

LeMma 3.6. Let $A$ be an element in a properly infinite von Neumann algebra $\mathbb{Q}$ with no o-finite type I direct summands and let $\left\{E_{n}\right\}$ be an infinite matrix base of $Q$ such that $\left\|E_{n} B E_{m}\right\|=0$ for $2 \leqslant m<n<\infty$ and $B$ in the set $\chi$ $=\left\{A^{*}, A, A^{*} A, A A^{*}\right\}$. Then there are orthogonal equivalent projections $E_{n 1}$ and $E_{n 2}$ of sum $E_{n}$ such that

$$
E_{11} A \sum\left\{E_{n 2} \mid n \geqslant 2\right\}=E_{12} A \sum\left\{E_{n 1} \mid n \geqslant 2\right\}=0 .
$$

Proof. Let $U$ be a partial isometry and $B$ a positive element of $Q$ so that $U B$ is the polar decomposition of $E_{1} A \sum\left\{E_{n} \mid n \geqslant 2\right\}$. Because each $E_{n}(n \geqslant 2)$ commutes with $B$ due to the validity of the relation

$$
E_{m} B^{*} B E_{n}=E_{m} A^{*}\left(E_{1}+E_{m}\right)\left(E_{n}+E_{1}\right) A E_{n}=E_{m} A^{*} A E_{n}=0
$$

for $2 \leqslant m<n<\infty$, each $E_{n}$ commutes with the range projection $E$ of $B$. There are equivalent orthogonal projections $F_{n 1}$ and $F_{n 2}(n \geqslant 2)$ commuting with $B$ and majorized by $E E_{n}$ such that

$$
F=\sum\left\{E E_{n}-\left(F_{n 1}+F_{n 2}\right) \mid n \geqslant 2\right\}
$$

is in the strong radical $g$ of $Q$ (Lemma 3.5). The projection $E_{n}-E_{n} E(n \geqslant 2)$ (resp. $E_{1}-U U^{*}$ ) is also the sum of orthogonal projections $G_{n 1}, G_{n 2}, G_{n 3}$ such that $G_{n 1} \sim G_{n 2}$ and $G_{n 3}$ is in $g$. Then we let

$$
E_{n 1}=\left(E E_{n}-F_{n 2}\right)+G_{n 1}+G_{n 3}
$$

and

$$
E_{n 2}=F_{n 2}+G_{n 2}
$$

for $n \geqslant 2$ and 


$$
E_{11}=U \sum\left\{E E_{n}-F_{n 2} \mid n \geqslant 2\right\} U^{*}+G_{11}+G_{13}
$$

and

$$
E_{12}=U \sum\left\{F_{n 2} \mid n \geqslant 2\right\} U^{*}+G_{12} \text {. }
$$

We have that $c\left(E_{n 1}\right)=c\left(E_{n 2}\right)=0$ for $n \geqslant 2$ and so $E_{n 1} \sim 1 \sim E_{n 2}$. Here $c=c_{g}$. Also we have that

$$
\begin{aligned}
c\left(E_{11}\right) & =c\left(U\left(F+\sum\left\{F_{n 1} \mid n \geqslant 2\right\}\right) U^{*}+G_{11}+G_{13}\right) \\
& =c\left(U\left(\sum\left\{F_{n 1} \mid n \geqslant 2\right\}\right) U^{*}+G_{11}\right) \\
& =c\left(U\left(\sum\left\{F_{n 2} \mid n \geqslant 2\right\}\right) U^{*}+G_{12}\right)=c\left(E_{12}\right),
\end{aligned}
$$

and thus $E_{11} \sim 1 \sim E_{12}$. We also have that

$$
E_{11} A \sum\left\{E_{n 2} \mid n \geqslant 2\right\}=U\left(E-\Sigma\left\{F_{n 2} \mid n \geqslant 2\right\}\right) \Sigma\left\{F_{n 2} \mid n \geqslant 2\right\} B=0
$$

and

$$
E_{12} A \sum\left\{E_{n 1} \mid n \geqslant 2\right\}=U \sum\left\{F_{n 2} \mid n \geqslant 2\right\}\left(E-\sum\left\{F_{n 2} \mid n \geqslant 2\right\}\right) B=0 .
$$

Q.E.D.

Now if 0 is in the essential central spectrum of $A$ in the properly infinite von Neumann algebra $Q$, then we may employ Theorem 2.10 and Lemma 3.6 (as applied to $\left.A^{*}\right)$ to write $A$ as a $4 \times 4$-matrix $\left(A_{i j}\right)$ over $\mathbb{Q}$ such that $A_{41}=A_{32}$ $=0$ and such that $A_{33}$ and $A_{44}$ can be further decomposed to infinite operator matrices $\left(C_{i j}\right)$ over $Q$ of the form $\left\|C_{i j}\right\| \leqslant \alpha_{i} \delta_{i j}$. To apply Lemma 3.6 to this new matrix we need the next lemma.

LEMMA 3.7. Let $A$ be an element of a properly infinite von Neumann algebra $\mathbb{Q}$ and let $\left\{F_{n}\right\}$ be an infinite matrix base for $Q$ such that $F_{1} A F_{m}=0$ for $m \geqslant 2$ and $\left\|F_{n} A F_{m}\right\| \leqslant \alpha_{n} \delta_{m n}$ for $m, n \geqslant 2$; then there is an infinite matrix base $\left\{E_{n}\right\}$ for $\mathbb{Q}$ such that $E_{n} B E_{m}=0$ for $2 \leqslant m<n<\infty$ and $B$ in the set $X=\left\{1, A, A^{*}\right.$, $\left.A A^{*}, A^{*} A\right\}$, and $\left\|E_{1} A E_{m}\right\| \leqslant \alpha_{m},\left\|E_{m} A E_{m}\right\| \leqslant \alpha_{m}$ for $m \geqslant 2$.

Proof. We use the same arguments as in the proof of Theorem 2.10. Let $\mathcal{S}$ be the family of all sets

$$
S=\left\{E_{n i} \mid(n, i) \in N \times I\right\}
$$

of nonzero projections in $\mathbb{Q}$ such that 
(i) $E_{m i} \sim E_{n i}$ for all $m, n$ in $N$ and $i \in I$;

(ii) $E_{n j} B E_{m i}=0$ for all $B$ in $\mathcal{X}$ and distinct pairs $(m, i),(n, j)$ in $N \times I$; and (iii) $E_{n i} \leqslant F_{n}$ for all $n$ in $N$ and $i$ in $I$.

From Lemma 2.9, we conclude that $\delta$ is nonvoid, and from Zorn's lemma we find a maximal set $S=\left\{E_{n i} \mid(n, i) \in N \times I\right\}$ in $\delta$ in the partial ordering (3). Let $\left\{N_{n} \mid n \in N\right\}$ be a partition of $N$ into a sequence of infinite sets such that the smallest element of $N_{n}$ is not less than $n$. As in Lemma 2.9, we can show that each of the projections

$$
E_{n}=\sum\left\{E_{m i} \mid(m, i) \in N_{n} \times I\right\}
$$

is a properly infinite projection of central support 1 . We show that each $E_{n}$ is equivalent to 1 . If $\mathscr{Q}$ is the product of $\sigma$-finite algebras, then each $E_{n}$ is equivalent to 1 since each $E_{n}$ is properly infinite. So we may assume that $\mathscr{Q}$ has no $\sigma$-finite direct summands. We show that $E_{n} \sim 1$ by showing that the largest central projection $P$ such that $P E_{n}$ is in the strong radical $g$ is $P=0$. We argue by contradiction. As in Theorem 2.10, we have that $P \sum E_{n}$ is in $g$. For every $n=1,2, \ldots$, the orthogonal complement $G_{n}$ in $P F_{n}$ of the least upper bound of the range supports of the elements $F_{n} B P \sum E_{n}$ for $B$ in $\mathcal{X}$ is equivalent to $P$ due to the fact this least upper bound is in $g$. Setting $G=\sum G_{n}$ and applying Lemma 2.9 to the element $G A G$ in the properly infinite algebra $\mathbb{Q}_{G}$ and to the sequence $\left\{G_{n}\right\}$ of projections, we may find a sequence $\left\{G_{n k}\right\}$ of projections in $\mathbb{Q}_{G}$ so that

$$
\left\{E_{n i} \mid(n, i) \in N \times(I \cup\{k\})\right\}
$$

is greater than $S$ in the partial ordering on $\delta$. This is a contradiction. So $E_{n} \sim 1$.

Replacing $E_{1}$ by $1-\sum\left\{E_{n} \mid n \geqslant 2\right\}$ if necessary, we obtain a matrix base $\left\{E_{n}\right\}$ that satisfies the required conditions. On the one hand, we have

$$
\left\|E_{m} A E_{m}\right\|=\operatorname{lub}\left\{\left\|E_{n i} A E_{n i}\right\| \mid(n, i) \in N_{m} \times I\right\} \leqslant \alpha_{m}
$$

for $m \geqslant 2$, and

$$
\left\|E_{m} B E_{n}\right\|=\operatorname{lub}\left\{\left\|E_{p i} B E_{q j}\right\| \mid(p, q, i, j) \in N_{m} \times N_{n} \times I \times I\right\}=0
$$

for $m, n \geqslant 2$ and $B$ in $\chi$. On the other hand, we have that 


$$
\begin{aligned}
\left\|E_{1} A E_{m}\right\| & \leqslant\left\|A \sum\left\{E_{n i} \mid(n, i) \in N_{m} \times I\right\}\right\| \\
& \leqslant\left\|\sum\left\{A \sum\left\{E_{n i} \mid i \in I\right\} \mid n \in N_{m}\right\}\right\| \leqslant\left\|\sum\left\{A F_{n} \mid n \in N_{m}\right\}\right\| \\
& \leqslant\left\|\sum\left\{F_{n} A F_{n} \mid n \in N_{m}\right\}\right\| \leqslant \operatorname{lub}\left\{\left\|F_{n} A F_{n}\right\| \mid n \in N_{m}\right\} \leqslant \alpha_{m}
\end{aligned}
$$

for $m \geqslant 2$. Q.E.D.

Now we can prove our main theorem.

THEOREM 3.8. An element $A$ in a properly infinite von Neumann algebra $\mathbb{Q}$ is a selfadjoint commutator if and only if 0 is in the essential central range of $A$ modulo the strong radical of $Q$.

Proof. If $A$ is a selfadjoint commutator, then the essential central spectrum $\mathcal{F}(A)$ of $\mathbb{Q}$ modulo the strong radical $g$ contains 0 . The proof presented in Theorem 3.4 is sufficient.

Conversely, we may assume $Q$ has no $\sigma$-finite type I direct summands (Theorem 3.4). Let $A$ be in $\mathbb{Q}$ and let $0 \in \mathcal{K}(A)$. We have already indicated after Lemma 3.5 that with regard to a matrix base $E_{i}(1 \leqslant i \leqslant 4)$ the operator $A$ can be written as a $4 \times 4$-matrix $\left(A_{i j}\right)$ over $\mathbb{Q}$ such that $A_{41}=A_{32}=0$, and such that $A_{33}$ and $A_{44}$ can be written with respect to an infinite matrix base as matrices over $\mathcal{Q}$ of the form $\left(C_{i j}\right)$, where $\left\|C_{i j}\right\| \leqslant \alpha_{j} \delta_{i j}$ for $i, j=1,2, \ldots$ Let $A^{\prime}$ (resp. $A^{\prime \prime}$ ) be given by

$$
A^{\prime}=\left(\begin{array}{cc}
A_{11} & A_{14} \\
0 & A_{44}
\end{array}\right)\left(\text { resp. } A^{\prime \prime}=\left(\begin{array}{cc}
A_{22} & A_{23} \\
0 & A_{33}
\end{array}\right)\right)
$$

Setting $C$ equal to $A^{\prime}$ (resp. $A^{\prime \prime}$ ) and applying Lemma 3.7 to $C^{*}$, we get a matrix base $\left\{F_{n} \mid 1 \leqslant n<\infty\right\}$ for $Q$ such that $F_{n} B F_{m}=0$ for all $2 \leqslant m<n$ $<\infty$ and $B$ in the set $\mathcal{X}=\left\{1, C, C^{*}, C^{*} C, C C^{*}\right\}$, and $\left\|F_{m} C F_{1}\right\| \leqslant \alpha_{m}$, $\left\|F_{m} C F_{m}\right\| \leqslant \alpha_{m}$ for $m \geqslant 2$. From Lemma 3.6 applied to the matrix base $\left\{F_{n}\right\}$, we see that $C$ may be written as a $4 \times 4$-matrix over $Q$ of the form Figure 1 , where $\left\|A_{i}\right\|,\left\|B_{i}\right\|,\left\|C_{i}\right\|,\left\|D_{i}\right\|$ are majorized by $\alpha_{i}$. Actually, the entries (*) of the 3,1 and 4,2 (resp. 3,4 and 4,3) blocks have the same form as the 4,1 (resp. $4,4)$ block. However, we do not need this information here. Reassembling the matrix $A$ from the various parts and applying J. Anderson's rearrangement [1], viz. $1 \rightarrow 7 \rightarrow 6 \rightarrow 4 \rightarrow 1,2 \rightarrow 5 \rightarrow 2,3 \rightarrow 3,8 \rightarrow 8$, we obtain an $8 \times 8$ matrix over $\mathcal{Q}$ whose four $2 \times 2$-diagonal blocks have the form Figure 2, where $\left\|B_{i}\right\| \leqslant \alpha_{i},\left\|C_{i}\right\| \leqslant \alpha_{i}$. From the calculation of M. David [5], we get that each of these four diagonal blocks is a selfadjoint commutator in $\mathbb{Q}$ and thus that $A$ itself is a selfadjoint commutator (cf. [1], [11, Proof, 4]). Q.E.D. 


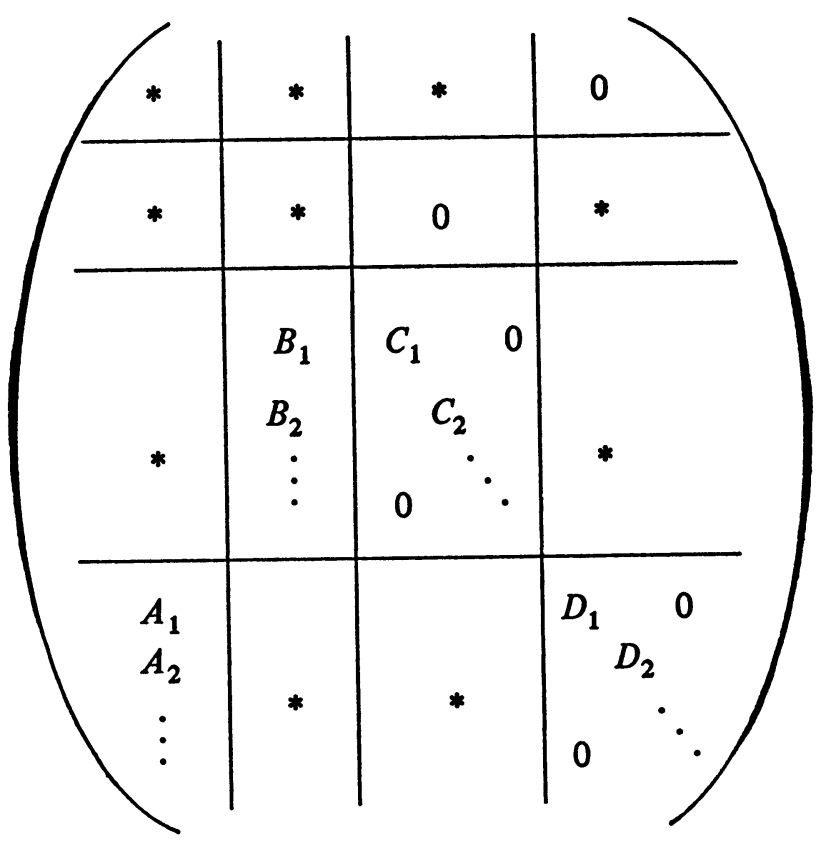

Figure 1

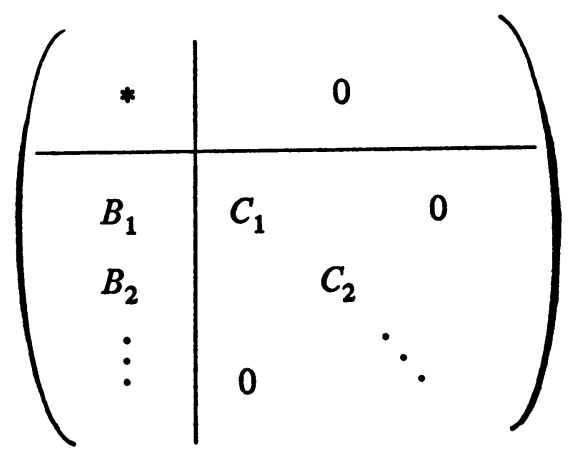

Figure 2

An operator $A$ in a von Neumann algebra $Q$ is said to be similar to an operator $B$ in $Q$ if there is an invertible $C$ in $Q$ with $C A C^{-1}=B$. We can now characterize those operators in a properly infinite algebra that are similar to a selfadjoint commutator. 
Proposition 3.9. An operator $A$ in a properly infinite von Neumann algebra $\mathbb{Q}$ with strong radical $g$ is similar to a selfadjoint commutator in $\mathbb{Q}$ if and only if there is $a \kappa>0$ such that

$$
\|(\alpha-A)(q+[\zeta])\| \geqslant \kappa|\alpha|
$$

for every complex $\alpha$ and every $\zeta$ in the spectrum $Z$ of the center $\mathcal{Z}$ of $\mathbb{Q}$, or equivalently, such that

$$
\|(\alpha-A)(\Re)\| \geqslant \kappa|\alpha|
$$

for every $\alpha$ and maximal ideal $\mathfrak{N}$ in $\mathbb{Q}$.

Proof. Suppose there exists such a $\kappa>0$. For convenience suppose that $\|A\|=1$. There is a $C$ in $\mathscr{Z}$ such that

$$
C^{\wedge}(\zeta)=\|A(g+[\zeta])\|
$$

$[12,3.2]$. There is a projection $P$ in $\mathcal{Z}$ such that $C P>0$ (i.e. $\left\{\zeta \in Z \mid C^{\wedge}(\zeta)\right.$ $>0\}$ is dense in $\left.\left\{\zeta \in Z \mid P^{\wedge}(\zeta)=1\right\}\right)$ and $C(1-P)=0$. We have that $A(1-P)$ is in the strong radical of $\mathbb{Q}_{(1-P)}[12,3.1]$, and thus $A(1-P)$ is a selfadjoint commutator in $\mathbb{Q}_{(1-P)}$. By passing to the direct summand $\mathfrak{Q}_{P}$, we may assume that $C>0$. There is an orthogonal sequence $\left\{P_{n}\right\}$ of projections in $\mathcal{Z}$ of sum 1 such that $C^{\wedge}(\zeta)$ is in the interval $\left[(n+1)^{-1}, n^{-1}\right]$ whenever $P_{n}^{\wedge}(\zeta)=1$. For every $n=1,2, \ldots$ there is a $B_{n}$ in $g$ such that

$$
\left\|\left(A+B_{n}\right)([\zeta])\right\|
$$

is in the interval $\left[(n+1)^{-1}, 2 n^{-1}\right]$ for every $\zeta$ with $P_{n}^{\wedge}(\zeta)=1$. The sum $\sum(n+1)\left(A+B_{n}\right) P_{n}$ converges in the strong topology to an element $B$ in $\mathbb{Q}$. We have that

$$
\|B(g+[\zeta])\| \geqslant 1
$$

and

$$
\|(\alpha-B)(g+[\zeta])\| \geqslant \kappa|\alpha|
$$

for every complex $\alpha$ and every $\zeta$ in the union of the sets $\left\{\zeta \in Z \mid P_{n}^{\wedge}(\zeta)=1\right\}$ and thus for every $\zeta$ in $Z$. This means that the canonical image of $B$ in the algebra $\mathbb{Q} /(\mathcal{G}+[\zeta])$ is never a scalar multiple of the identity. There is an invertible $C$ in $Q$ and a projection $F$ equivalent to 1 such that $F C B C^{-1} F=0$ [11, Proof, Theorem 4]. For every $\varepsilon>0$ and every $n=1,2, \ldots$, there is a projection $E_{n}$ in $g$ such that

$$
\left\|B_{n}\left(1-E_{n}\right)\right\| \leqslant \varepsilon /\left(\|C\|\left\|C^{-1}\right\|\right) .
$$


The range projection $F_{n}$ of $F\left(C^{-1}\right)^{*} E_{n}$ is in $g$ and thus $G_{n}=F-F_{n}$ is equivalent to 1 . Setting $G=\sum G_{n} P_{n}$, we get a projection $G$ equivalent to 1 such that

$$
\left\|G C A C^{-1} G\right\| \leqslant\left\|F C B C^{-1} F\right\|+\|C\|\left\|C^{-1}\right\| \operatorname{lub}_{n}\left\|B\left(1-E_{n}\right)\right\| \leqslant \varepsilon .
$$

We conclude that $\mathscr{K}\left(C A C^{-1}\right)$ contains 0 (Theorem 2.2), and consequently that $C A C^{-1}$ is a selfadjoint commutator in $\mathcal{Q}$ (Theorems 3.4 and 3.8).

Conversely, let $A$ be a selfadjoint commutator in $Q$. We have that

$$
\|(\alpha-A)(g+[\zeta])\| \geqslant|\alpha|
$$

for every scalar $\alpha$ and every $\zeta \in Z$; otherwise,

$$
|\alpha|=\left|\phi(\alpha-A)^{\wedge}(\zeta)\right| \leqslant\|(\alpha-A)(\xi+[\zeta])\|<|\alpha|
$$

for some $\alpha$ and $\zeta$. Here $\phi$ is a state of $A^{\sim}$ which vanishes on $g \cup\{A\}$ (Theorems 3.4 and 3.8). If $C$ is an invertible element in $Q$, then we have that

$$
\left\|\left(\alpha-C A C^{-1}\right)(g+[\zeta])\right\| \geqslant\left(\|C\|\left\|C^{-1}\right\|\right)^{-1}|\alpha|
$$

for every $\alpha$ and $\zeta$. Q.E.D.

REMARK. The preceding proposition shows that the operators of class $\left(F^{\prime}\right)$ in a properly infinite algebra, viz. those operators which are not equal to a scalar multiple of the identity (zero included) modulo any maximal ideal, that were studied in [10] are contained in the set of operators similar to a selfadjoint commutator.

COROLLARY 3.10. For any operator $A$ and any normal operator $B$ in a properly infinite von Neumann algebra the operator $B A-A B$ is a selfadjoint commutator.

Proof. We may assume that 0 is in the essential central spectrum of $B$ modulo the strong radical $[12,3.5]$. For every $\varepsilon>0$, there is a projection $E$ in $\mathcal{Q}$ equivalent to the identity with

$$
\|E B\|=\|B E\| \leqslant \varepsilon
$$

(Corollary 2.6). Hence, the essential central spectrum of $B A-A B$ modulo the strong radical contains 0 (Theorem 2.2) and so $B A-A B$ is a selfadjoint commutator (Theorem 3.8). Q.E.D.

REMARK. Actually, for any $A$ and any seminormal $B$ (i.e. either $\pm\left(B B^{*}-B^{*} B\right)$ is positive) in a properly infinite algebra, the operator $A B-B A$ is a selfadjoint commutator (cf. [11, Note "added in proof"]).

The next proposition extends the results of $H$. Radjavi [13] for the algebra of bounded operators on a separable Hilbert space. 
Proposition 3.11. A selfadjoint operator in a properly infinite von Neumann algebra is a selfadjoint commutator if and only if its essential central spectrum modulo the strong radical contains a nonpositive and a nonnegative element.

PROof. The essential central range of a selfadjoint operator modulo the strong radical is the smallest central convex set containing the essential central spectrum modulo the strong radical $[12,4.4]$. Q.E.D.

The next result extends that of J. Williams [14] for the algebra of all bounded operators on a separable Hilbert space.

PROPOSITION 3.12. Every commutator of a properly infinite factor von Neumann algebra is similar to a selfadjoint commutator.

Proof. An operator $A$ is a commutator in a properly infinite factor $\mathbb{Q}$ if and only if there is an invertible $B$ in $\mathbb{Q}$ and a projection $E$ in $\mathbb{Q}$ with $E \sim 1$ such that $E B A B^{-1} E=0$ [3], [4], [10]. Thus, every commutator of $\mathcal{Q}$ is similar to a selfadjoint commutator (Theorems 3.4 and 3.8).

One may also use the arguments of J. Williams [14] together with Theorems 3.4 and 3.8 to avoid the computations of [3], [4], [10]. Q.E.D.

One might conjecture that an operator is a commutator if and only if it is not equal to a nonzero scalar multiple of the identity modulo every maximal ideal (i.e. if and only if it is an operator of class $(F)$ ). If the center is large, this turns out to be false.

EXAMPLE 3.13. In every properly infinite von Neumann algebra $\mathbb{Q}$ not equal to a finite product of factors, there is an operator of class $(F)$ which is not a commutator. Indeed, there is for every natural number $m$ a neighborhood $V=V_{m}$ of 1 contained in the sphere of radius one about 1 such that $A B-B A$ in $V_{m}$ and $\|A\| \leqslant 1$ implies $\|B\| \geqslant m$. This holds even for factors. Notice that every neighborhood of the identity contains commutators [10,3.10]. On the contrary, there would be bounded sequences $\left\{A_{n}\right\}$ and $\left\{B_{n}\right\}$ in $\mathbb{Q}$ with $\left\{A_{n} B_{n}-B_{n} A_{n}\right\}$ converging to the identity. This is impossible [16, Problem 183]. So such a neighborhood $V_{m}$ exists.

Now let $\left\{P_{m}\right\}$ be an orthogonal sequence of nonzero central projections of $\mathbb{Q}$ of sum 1. There is a commutator $C_{m}$ in $Q P_{m}$ such that $\left\|C_{m}-1\right\| \leqslant 1$ and $C_{m}=A B-B A$ for $\|A\| \leqslant 1$ implies $\|B\| \geqslant m^{2}$. Then the operator $C$ $=\sum m^{-1} C_{m}$ is in class $(F)$. In fact, let $\zeta$ be in the spectrum of the center. Either $P_{m}^{\wedge}(\zeta)=1$ for some $m$ or $P_{m}^{\wedge}(\zeta)=0$ for all $m$. In the first case

$$
C(g+[\zeta])=(B A-A B)(g+[\zeta])
$$

for some $A$ and $B$ in $Q P_{m}$. Here $g$ is the strong radical. Hence $C$ is not a nonzero scalar multiple of the identity modulo $g+[\zeta]$. In the second case we have that 


$$
\|C(g+[\zeta])\| \leqslant\left\|C\left(1-\sum\left\{P_{n} \mid n \geqslant m\right\}\right)([\zeta])\right\| \leqslant 2(m+1)^{-1}
$$

and so $C(\xi+[\zeta])=0$. Now suppose $C=A B-B A$ for $A, B$ in $Q$. We may assume that $\|A\| \leqslant 1$. This means that $\left\|m B P_{m}\right\| \geqslant m^{2}$. This is impossible. So $C$ is not a commutator.

In contradistinction to factors we show that not every commutator is similar to a selfadjoint commutator when the center is sufficiently large.

Proposition 3.14. Let $A$ be an operator of class $(F)$ in a properly infinite von Neumann algebra $\mathbb{Q}$; then there is a sequence $\left\{\beta_{n}\right\}$ of strictly positive numbers and a sequence $\left\{P_{n}\right\}$ of orthogonal central projections of sum 1 such that $\sum \beta_{n} A P_{n}$ converges in the strong topology to a commutator in $\mathbb{Q}$.

Proof. Let $g$ be the strong radical of $\mathbb{Q}$ and let $P_{0}=c_{g}(A)$. There is a sequence $\left\{P_{n} \mid n \geqslant 1\right\}$ of orthogonal central projections of sum $1-P_{0}$ such that $\|A(g+[\zeta])\| \geqslant n^{-1}$ for every $\zeta$ in the spectrum of the center of $Q P_{n}[12,3.1]$. Therefore, the operators $A P_{n}(n \geqslant 1)$ are of class $\left(F^{\prime}\right)$ in $Q P_{n}$. There is $B_{n}$ and $C_{n}$ in $Q P_{n}$ with

$$
A P_{n}=B_{n} C_{n}-C_{n} B_{n}
$$

for all $n \geqslant 0$ [10]. Setting $\beta_{n}$ equal to the inverse of $\left\|B_{n}\right\|\left\|C_{n}\right\|+1$, we see that $\sum \beta_{n} A P_{n}$ converges in the strong topology and that it is a commutator in $\mathbb{Q}_{\text {. }}$ Q.E.D.

EXAMPLE 3.15. Let $\mathcal{Q}$ be a properly infinite von Neumann algebra. Suppose there is a sequence $\left\{P_{n}\right\}$ of nonzero orthogonal central projections of sum 1. Let $E_{n}$ be a projection in $Q P_{n}$ such that $E_{n} \sim P_{n}-E_{n} \sim P_{n}$ and let

$$
A=\sum\left(n^{-1} P_{n}+n^{-2} E_{n}\right) .
$$

Then $A$ is in class $(F)$ and so there is a sequence $\left\{\beta_{n}\right\}$ of strictly positive numbers and a sequence $\left\{Q_{n}\right\}$ of orthogonal central projections of sum 1 such that $B=\sum \beta_{n} A Q_{n}$ is a commutator in $Q$ (Proposition 3.14). There is no state $\phi$ in $\mathbb{Q}^{\sim}$ and invertible $C$ in $\mathbb{Q}$ with $\phi(\mathcal{g})=(0)$ and $\phi\left(C B C^{-1}\right)=0$; otherwise, the state $\phi$ would vanish on $C A C^{-1}$ or equivalently $\mathscr{K}\left(C A C^{-1}\right)$ would contain 0 . This is known to be impossible (cf. [11, p. 63ff.]). Hence, the operator $B$ is not similar to a selfadjoint commutator.

\section{BIBLIOGRAPHY}

1. Joel Anderson, Derivations, commutators and the essential numerical range, Thesis, Indiana Univ., 1971.

2. Joel Anderson and J. G. Stampfli, Commutators and compressions, Israel Math. J. 10 (1971), 433-441. MR 47 \#874. 
3. A. Brown and C. Pearcy, Structure of commutators of operators, Ann. of Math. (2) 82 (1965), 112-127. MR 31 \#2612.

4. - Commutators in factors of type III, Canad. J. Math. 18 (1966), 1152-1160. MR 34 $\# 1864$.

5. M. David, On a certain type of commutator, J. Math. Mech. 19 (1969/70), 665-680. MR 41 \#2437.

6. D. Deckard and C. Pearcy, On continuous matrix-valued functions on a Stonian space, Pacific J. Math. 14 (1964), 857-869. MR 30 \#2356.

7. J. Diximier, Les algèbres d'opérateurs dans l'espace hilbertien (Algèbres de von Neumann), Gauthier-Villars, Paris, 1957. MR 20 \# 1234.

8. J. G. Glimm, A Stone-Weierstrass theorem for $C^{*}$-algebras, Ann. of Math. (2) 72 (1960), 216-244. MR 22 \#7005.

9. H. Halpern, Irreducible module homomorphisms of a von Neumann algebra into its center, Trans. Amer. Math. Soc. 140 (1969), 195-221. MR 39 \#3322.

10. - Commutators in properly infinite von Neumann algebras, Trans. Amer. Math. Soc. 139 (1969), 55-73. MR 40 \#4773.

11. Commutators modulo the center in a properly infinite von Neumann algebra, Trans. Amer. Math. Soc. 150 (1970), 55-68. MR 43 \#2526.

12. - Essential central spectrum and range for elements of a von Neumann algebra, Pacific J. Math. 43 (1972), 349-380. MR 48 \#2787.

13. H. Radjavi, The structure of $A^{*} A-A A^{*}$, J. Math. Mech. 16 (1966), 19-26. MR 34 \#3332.

14. J. P. Williams, On commutativity and the numerical range in Banach algebras, J. Functional Analysis 10 (1972), 326-329. MR 50 \# 14229.

15. L. Zsidó, Note on Dixmier's trace type sets in properly infinite $W^{*}$-algebras, Rev. Roumaine Math. Pures Appl. 19 (1974), 269-274. MR 49 \#9643.

16. P. R. Halmos, A Hilbert space problem book, Van Nostrand, Princeton, N. J., 1967. MR 34 \#8178.

Department of Mathematics, University of Cincinnati, Cincinnati, Ohio 45221 